\title{
Turbulence-flame interactions in lean premixed hydrogen: transition to the distributed burning regime
}

\author{
A. J. ASPDEN†, M. S. DAY AND J. B. BELL \\ Center for Computational Sciences and Engineering, Lawrence Berkeley National Laboratory, \\ 1 Cyclotron Road, MS 50A-1148, Berkeley, CA 94720, USA
}

(Received 26 February 2010; revised 15 March 2011; accepted 1 April 2011;

first published online 31 May 2011)

The response of lean $(\varphi \leqslant 0.4)$ premixed hydrogen flames to maintained homogeneous isotropic turbulence is investigated using detailed numerical simulation in an idealised three-dimensional configuration over a range of Karlovitz numbers from 10 to 1562. In particular, a focus is placed on turbulence sufficiently intense that the flames can no longer be considered to be in the thin reaction burning regime. This transition to the so-called distributed burning regime is characterised through a number of diagnostics, and the relative roles of molecular and turbulent mixing processes are examined. The phenomenology and statistics of these flames are contrasted with a distributed thermonuclear flame from a related astrophysical study.

Key words: flames, turbulence simulation, turbulent reacting flows

\section{Introduction}

There has been considerable recent interest in the development of lean premixed combustors capable of burning hydrogen or hydrogen-rich mixtures obtained from gasification of coal or biomass as alternatives to petroleum. Burning under lean conditions reduces the exhaust gas temperatures and consequently thermal $\mathrm{NO}_{x}$ emissions. Burning pure hydrogen-air mixtures have the additional advantage of avoiding carbon chemistry altogether, thereby producing zero carbon dioxide in the burner. In practical devices, turbulent intensities can be extremely high, and Strakey, Sidwell \& Ontko (2007) suggest that root mean square (r.m.s.) velocity fluctuations can reach as high as 150 times the unstretched laminar flame speed. There is little work in the literature that deals with flames subjected to such high-intensity turbulence. Here, we present three-dimensional numerical simulations that explore turbulenceflame interactions of lean premixed hydrogen over a range of turbulent intensities up to approximately 100 times the unstretched laminar flame speed, with a focus on examining the transition to 'distributed burning'.

To give context for the discussion of the distributed burning regime, we first present a summary of the development of regime diagrams that have been used to characterise turbulent premixed flames since their introduction by Borghi (1985), 

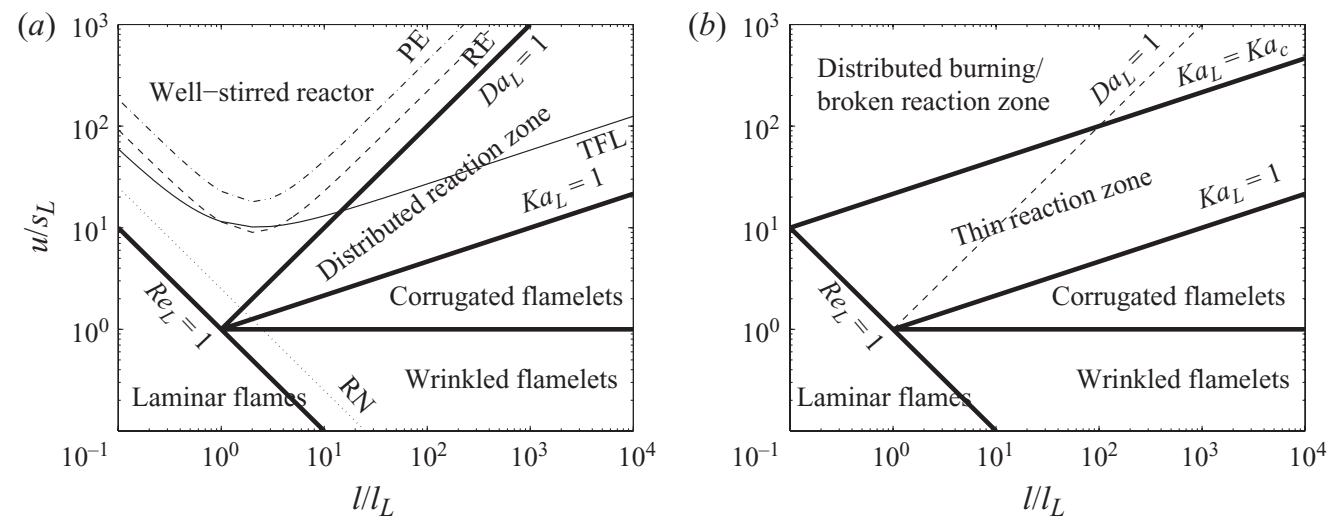

FIGURE 1. Regime diagrams following $(a)$ in its original from Peters (1986) and $(b)$ modified following Peters $(1999,2000)$ and including the distributed burning regime for $K a_{L}>K a_{c}$ (here we have used $K a_{c}=K a_{\delta}$ with $\left.l_{\delta}=l_{L} / 10\right)$. In $(a)$, the lines RE and PE approximate the extinction lines according to Roberts et al. (1993) and Poinsot et al. (1990), respectively. TFL denotes the thin flamelet limit, $\mathrm{RN}$ line is the region of no effect, both from Roberts et al. (1993).

Williams $(1985 a, b)$ and Peters (1986). Three important dimensionless groups used for this characterisation are the flame Reynolds, Karlovitz and Damköhler numbers, respectively, defined to be

$$
R e_{L}=\frac{\check{u} l}{s_{L} l_{L}}, \quad K a_{L}^{2}=\frac{\check{u}^{3} l_{L}}{s_{L}^{3} l}, \quad D a_{L}=\frac{s_{L} l}{\check{u} l_{L}},
$$

where $\check{u}$ and $l$ are the turbulent intensity and integral length scale, respectively, and the flame propagation speed $s_{L}$ and thermal thickness $l_{L}=\Delta T / \max (|\nabla T|)$ are defined for a one-dimensional unstretched steady laminar flame. Note that for the order-of-magnitude purposes appropriate for the regime diagram, no distinction is made between the different diffusion coefficients, which explains the form of the flame Reynolds number, and means that only two of the three dimensionless quantities in (1.1) are independent $\left(K a_{L}^{2} D a_{L}^{2}=R e_{L}\right)$.

The first regime diagrams (figure 1a) used the Klimov-Williams criterion, i.e. $K a_{L}=1$ (see Klimov 1963; Williams 1976), to distinguish between the so-called flamelet regime (defined for $R e_{L}>1$ and $K a_{L}<1$ ) and distributed reaction zone regime (defined there for $R e_{L}>1, K a_{L}>1$ and $D a_{L}>1$ ), see Peters (1986) in particular. The argument was that for $K a_{L}>1$ the Kolmogorov length scale $\eta$ is smaller than the flame width so that the smallest scales of turbulence can enter the laminar flame structure. Pope (1987) proposed an intermediate regime citing Abraham, Williams \& Bracco (1985) to argue that $K a_{L}<1$ is a 'sufficient condition for flame-sheet combustion, but it may not be necessary'.

Based on two-dimensional simulations of vortex-flame interaction, Poinsot, Veynante \& Candel (1990) suggested that the distributed reaction zones regime would take an order of magnitude more intense turbulence, see also Poinsot, Veynante \& Candel (1991); Meneveau \& Poinsot (1991), and the experimental study of single vortex-flame interactions by Roberts et al. (1993). In both the numerical and experimental studies, a 'quenching zone' was identified where the flame was quenched by the flow, and 'no effects zone', where flow strain even at the integral scale is unable to impact the internal flame structure significantly.

A series of papers by Mansour, Chen \& Peters (1992), Chen et al. (1996), Chen $\&$ Mansour (1997) and Mansour, Peters \& Chen (1998) looked at highly stretched 
premixed methane Bunsen flames at high Karlovitz numbers. As the exit velocity was increased, local extinction events became more frequent, until eventually global extinction occurred. Based on these observations, it has been argued (e.g. Peters 2000) that turbulence-enhanced heat losses will lead to global quenching of the flame, and therefore, a premixed flame cannot survive at such high Karlovitz numbers. Peters $(1999,2000)$ presented a modified regime diagram (see figure $1 b$ ), where the reaction zone thickness was used to define a critical Karlovitz number as the limit of the flamelet regime. Specifically, taking the reaction zone thickness to be $l_{\delta} \approx l / 10$, then $K a_{\delta}^{2}=\left(\check{u}^{3} \delta\right) /\left(s_{L}^{3} l\right)=1$ gives $K a_{L} \approx 100$. The regime for $K a_{L}>100$ was renamed as the 'broken reaction zone', and the distributed reaction zone regime was eliminated.

In this paper, we present simulations that argue in favour of retaining the distributed reaction zone regime, in addition to the broken reaction zone. We contend that both kinds of flame can exist at high Karlovitz numbers, depending on the configuration of the flow. In the single vortex-flame interaction the flow geometry is a key element to the evolution of the flame. It is also likely to play a role in the stretched Bunsen flames, where the flame is also exposed to a cold environment. We argue that, under certain conditions, it is possible for high-Karlovitz-number flames to exist without extinguishing. Such possibilities may arise if the flames are embedded in fully developed turbulence, confined in some way and/or exposed to a hot environment.

Summerfield et al. $(1954,1955)$ were the first to use the term 'distributed reaction' referring to a flame where 'reaction rate laws and transport processes are modified by the presence of turbulence'. Damköhler (1940) identified a limiting behaviour referred to as 'the small-scale turbulence', bounded by $l / l_{L}<1$, and suggested scaling laws analogous to laminar flames with molecular diffusion replaced by turbulent diffusion. Williams (1985a) presented both the extinction arguments as above and also considered the limiting behaviour of the 'well-stirred reactor', where turbulent mixing is rapid compared with chemistry, thereby causing combustion to occur in a distributed reaction zone, "with heat release occurring more or less homogeneously throughout the turbulent flame brush and with local fluctuations in temperature and composition being small'. Bray (1995) refers to the distributed reaction regime where chemical times are long compared with the largest time scales of the turbulent flow, i.e. $D a_{L} \ll 1$, and suggests that this could be the mode of combustion in the rocket exhaust of Kalghatgi, Cousins \& Bray (1981). Pope \& Anand (1985) considered 'distributed combustion, in which reaction is distributed more uniformly in space and is not necessarily accompanied by steep spatial concentration gradients', and indicated that it should occur for $l / l_{L} \ll 1$.

All of these discussions are descriptions of the same type of flame behaviour and, thus, pertain to the same regime, which we will refer to as the distributed burning regime, and argue that it is bounded by $K a_{L}>K a_{c}$ for some critical Kalovitz number (to be discussed below). For fully developed turbulence, the Karlovitz number quantifies the intensity of turbulence that occurs at length scales comparable to the (internal) flame thickness, independent of the integral length scale (provided $l>l_{\delta}$ ). Specifically, for a particular flame (i.e. fixed $s_{L}$ and $l_{L}$ ) and a constant Karlovitz number, changing the integral length scale implies a change in turbulent intensity in such a way that the energy dissipation rate $\varepsilon=\breve{u}^{3} / l$ is fixed. Therefore, assuming a Kolmogorov turbulent cascade, the intensity of the turbulent eddies comparable in size with the flame (or the reaction zone thickness) remains unchanged. The consequence for the regimes of turbulent premixed flames is that the Karlovitz number is the appropriate quantity to differentiate between regimes affected by small-scale turbulence-flame interaction, in particular preference to the Damköhler 
number, which is more appropriate for large-scale interactions. Indeed, Damköhler's small-scale turbulence limit corresponds to large Karlovitz numbers, and the largescale turbulence limit corresponds to large Damköhler numbers. Following Peters (1999, 2000), $K a_{L}=1$ separates the corrugated and wrinkled flamelet regimes (where $l_{\delta}<l_{L}<\eta$ ) from the thin reaction zones regime (where $l_{\delta}<\eta<l_{L}$ ). In the corrugated flamelet regime, the Gibson scale $l_{G}=s_{L}^{3} / \varepsilon$ measures the size of turbulent eddy with speed equal to $s_{L}$. In the thin reaction zone, $l_{G}$ becomes smaller than $l_{L}$ so turbulent mixing can modify the flame structure on length scales comparable to the thermal thickness. In this regime, the reaction zone, which is typically an order of magnitude thinner and located downstream of the preheat zone, is not affected appreciably by the turbulence. With further increases in $K a_{L}$, turbulent mixing at the smaller length scales eventually becomes sufficiently energetic to disrupt the internal structure of the reaction zone. The latter condition marks the transition to distributed burning with the condition $K a_{\delta}=K a_{c}$ separating the thin reaction zone regime from the distributed burning and broken reaction zones regimes. The specific value of $K a_{c}$ is unknown, but one argument is that when $K a_{L}>K a_{\delta}$, the Kolmogorov length scale becomes smaller than the reaction zone thickness, i.e. where $\eta<l_{\delta}<l_{L}$.

There is little evidence of distributed flames in the literature that meet our criterion; however, the piloted premixed jet burner of Dunn, Masri \& Bilger (2007); Dunn et al. (2009) does provide one such example. Jet exit speeds between 50 and $200 \mathrm{~m} \mathrm{~s}^{-1}$ gave rise to turbulent intensities between 40 and 390 times $s_{L}$ and Karlovitz numbers between 100 and 3500. The Karlovitz 100 flame was argued to be flamelet-like, but the more turbulent cases were classified as distributed. Turbulence was shown to significantly disrupt and broaden the flame structures (in particular lower temperature gradients were observed with increased turbulent intensity). There was also evidence of extinction, especially at the highest intensity, but this is likely to be strongly affected by the mean flow. A relevant numerical study was conducted by Poludnenko \& Oran $(2010 a, b)$, who considered stoichiometric hydrogen-air flames with simple chemistry in high levels of turbulence $\left(K a_{L} \approx 60\right)$ using an approach closely related to the present study. They reported that subsonic turbulence could only broaden the preheat zone and did not influence the internal flame structure. Lean hydrogen burns with a slower and broader flame, and will therefore require lower turbulent intensities to make the transition to distributed burning.

Aspden et al. (2008a) examined turbulence-flame interactions in thermonuclear carbon-burning flames typical of type Ia supernovae at Karlovitz numbers up to 230 . Despite the disparate settings, the study is relevant to the present discussion. The burning is due to nuclear fusion, two carbon atoms combine to form magnesium; hence, the fuel can be considered to be premixed (and essentially stoichiometric as no oxidiser is required). The reaction kinetics were represented by a single-step thermonuclear reaction without intermediate species. Before any potential transition to detonation takes place, the speed of sound in the star far exceeds that of both the turbulence and flame propagation; the flow is low Mach number (indeed, much lower than any simulation presented here). Flame propagation is driven by thermal diffusion (due to optically thick radiation), but species diffusion and viscosity are negligible, i.e. the Lewis number is very large. This provides an interesting contrast to the low-Lewis-number hydrogen flames in the present study. A significant difference is due to the non-ideal equation of state, which includes significant components due to degenerate electrons and radiation pressure. However, the remaining components of the low-Mach-number flow model and turbulence-flame interaction regime are 
(a)

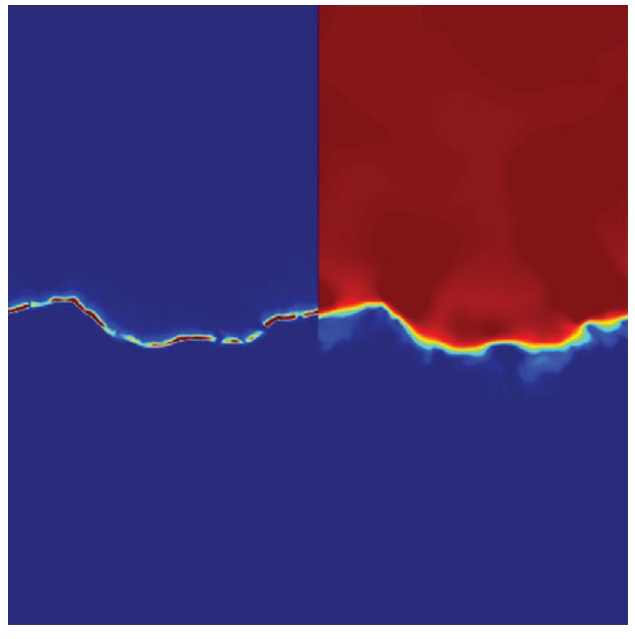

(c) $\left(\times 10^{6}\right)$

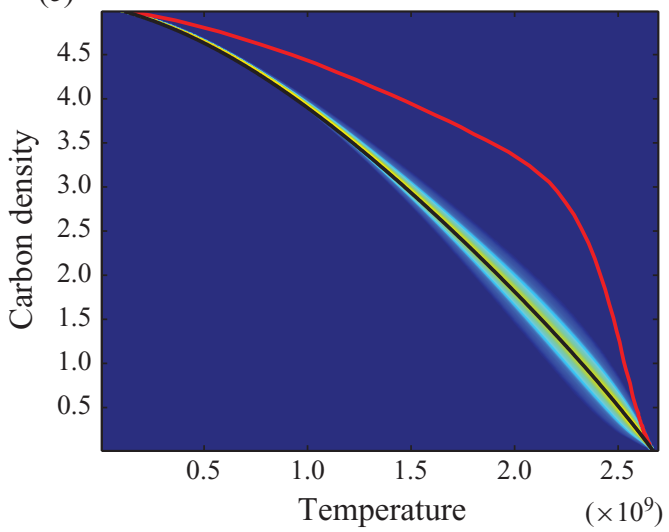

(b)

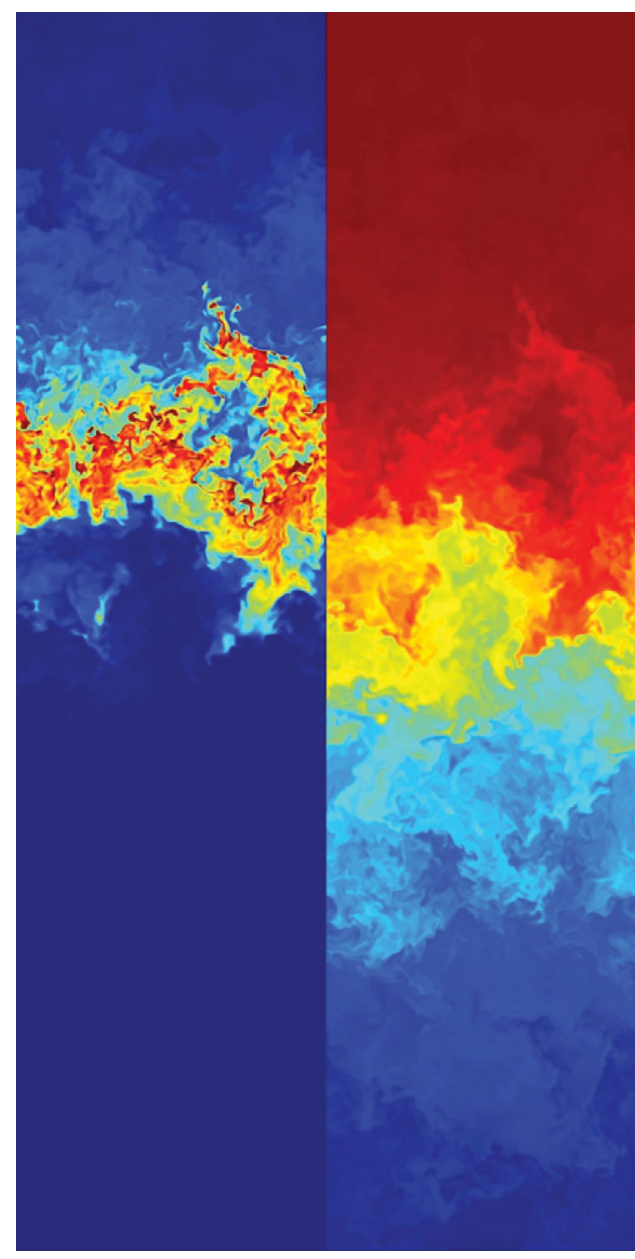

Figure 2. $(a, b)$ Two-dimensional slices from three-dimensional simulations of low- and high-Karlovitz-supernovae flames, respectively. The left-hand panel in each case is burning rate, and the right-hand panel is temperature. (c) Joint probability density function of fuel and temperature for the high Karlovitz case. The red line denotes the unstretched laminar flame profile and the black line denotes the burning profile without thermal or species diffusion.

relevant to the present study, and many parallels will be drawn to exposit the characterisation of the distributed burning regime.

We briefly summarise the relevant results of Aspden et al. (2008a) for context and partial motivation for the present study. The approach that was used will also be followed closely here. A downward-propagating flame was embedded in maintained homogeneous isotropic turbulence in a high-aspect-ratio domain with periodic lateral boundary conditions, a solid base, and outflow at the top. Figures 2(a) and $2(b)$ show two-dimensional slices through the three-dimensional simulations at $K a_{L}=0.1$ and 230, respectively. In each case, the left-hand panel shows the burning rate (blue is low and red is high) and the right-hand panel shows temperature (blue is the cold fuel temperature and red is the hot product temperature). At low-to-moderate 
Karlovitz numbers, $K a_{L} \lesssim 3$, turbulence disrupted the flame surface, an example of which is shown in figure 2(a), producing both regions of local extinction and regions of enhanced burning. The resulting turbulent flames were shown to propagate at a speed comparable to $s_{L}$. However, at a Karlovitz number of approximately 230 , the behaviour was categorically different. The flame was greatly broadened by the turbulence and there was no clear flame surface (see figure $2 b$ ). The peak burning rate was around one fifth of the unstretched laminar flame, but because a significantly greater volume of fluid was burning, the overall flame speed was approximately five or six times $s_{L}$. Joint probability density functions (JPDFs) of temperature and carbon density revealed that a significant shift in the burning behaviour had occurred. Figure 2(c) shows such a JPDF for the $K a_{L}=230$ case, including the distribution from the unstretched laminar flame (the red curve) and that of fuel burning isobarically with no thermal or species diffusion (the black curve). In the low turbulence cases (see figure 11 in Aspden et al. 2008a), a distribution close to the unstretched laminar flame was observed. At intermediate levels, the temperature and carbon density became decorrelated due to a competition between turbulent mixing and thermal conduction. At the highest Karlovitz number (see figure $2 c$ ), the distribution collapsed to one of an effectively unity Lewis number flame-turbulencedominated thermal diffusion; fuel and temperature were effectively mixed at the same rate. Aspden, Bell \& Woosley (2010) demonstrated that the small-scale turbulence theory of Damköhler (1940) could be successfully applied to these distributed flames at length scales spanning several orders of magnitude, confirming that the bound on distributed burning should be based on a critical Karlovitz number, rather than Damköhler number or length-scale ratio $\left(l / l_{L}\right)$.

We use a similar approach to examine the response of turbulent lean premixed hydrogen to such intense turbulence and compare low- and highLewis-number distributed flames. Besides the potential environmental benefits, hydrogen combustion can be well-approximated with a relatively small reaction set, which makes simulations computationally inexpensive, especially compared with larger hydrocarbons. Furthermore, the low Lewis number provides an interesting counterpoint to the high-Lewis-number supernovae flames. However, this low Lewis number makes lean premixed hydrogen-air flames thermodiffusively unstable (see, for example, Zeldovich 1944; Markstein 1949). Consequently, these flames propagate in cells of intense burning separated by regions of complete extinction. Therefore, even in the absence of turbulence, these flames are naturally wrinkled and highly nonuniform.

These cellular burning patterns have been experimentally observed in lean hydrogen by many researchers (see, for example, Bregeon, Gordon \& Williams 1978; Mitani \& Williams 1980). Turbulent lean hydrogen flames have also been studied computationally in both idealised configurations by Baum et al. (1994), Bell et al. (2007a) and Day et al. (2009a) and in a low-swirl burner configuration by Day et al. (2009b) and Bremer et al. (2009). The latter two papers show that for the moderate levels of turbulence characteristic of laboratory-scale flames, the effect of increasing turbulence is to reduce the size of cellular structures in the flame while increasing the variability of the local combustion intensity. This enhanced variability in intensity leads to local consumption rates of more than 40 times those of the idealized one-dimensional steady unstretched laminar flame for turbulent intensities of around $1 \mathrm{~m} \mathrm{~s}^{-1}$. (We note that for lean hydrogen flames, the notion of a steady unstretched flame is a theoretical construct; such a flame cannot be realized physically because of the thermodiffusive instability of the flame.) Another consequence of the 
increased variability of the combustion intensity is that the clear separation between the burning and non-burning regions along the flame front that is apparent at low levels of turbulence is highly blurred at these moderate levels. In practical devices, turbulent intensities can be as much as 50 times higher than those of the previous studies (see e.g. Strakey et al. 2007). It is perhaps reasonable to expect that under such conditions, turbulent transport will completely dominate molecular effects. However, it remains unclear how to characterise the transition to such a mode, and therefore to predict with any certainty the dynamics of flames into and approaching this regime. To date, there have been very few studies of combustion of lean $\mathrm{H}_{2}$-air mixtures in extremely intense turbulence, and no direct numerical simulation (DNS) investigations have been reported.

Given the potential benefits of successfully utilising pure lean hydrogen-air fuels, the aim of the present study is to explore the evolution of premixed hydrogen flames interacting with turbulence over a broad range of turbulent intensities, including values large enough to achieve burning in the distributed regime. The study is carried out using high-resolution three-dimensional simulations in an idealised configuration and incorporates detailed models for differential species transport and fundamental reaction kinetics. The study represents a first step in building a characterisation of the turbulence-flame interaction for non-unity Lewis number fuels in premixed combustion spanning flamelet to distributed regimes. A low-Machnumber combustion solver is used, which will be described in $\S 2$, using an idealised configuration described in $\S 3$. A range of equivalence ratios are considered from $\varphi=0.31$ to $\varphi=0.4$, where $\varphi=2 \xi_{\mathrm{H}} / \xi_{\mathrm{O}}$, and $\xi_{\mathrm{E}}$ denotes the molar concentration of element E. This range of equivalence ratios is typical of those used in experiments, e.g. Littlejohn \& Cheng (2007), because it is above the lean blow-off limit and below an equivalence ratio where flashback is a concern. We first examine the effect of Karlovitz number on the overall flame phenomenology. In $\$ 4.1 .2$, we present a sequence of simulations at an equivalence ratio of $\varphi=0.31$ over a range of Karlovitz numbers ranging from 10, which is in the thin reaction zone regime, up to 1562 , which is much higher than the distributed supernova flame $\left(K a_{L}=230\right)$. Despite this, it will be shown that the most turbulent flame does not appear to be burning in a distributed mode. We then present a similar sequence of simulations for $\varphi=0.40$ in $\$ 4.1 .3$. In this case, it will be shown that the highestKarlovitz-number flame does appear to be distributed. We use PDFs of density gradients $(|\nabla \rho|)$ to examine the extent of turbulent mixing on the flame structure. The distribution decays rapidly for flames in the thin reaction zone regime but transitions to an exponential distribution typical of a turbulent mixing layer when the flame becomes distributed. To examine the interaction of turbulence with the flames in more detail, we computed the conditional mean and variance of temperature as a function of fuel mass fraction (similar to the JPDF presented in figure $2 c$ for the distributed supernova flame), and local equivalence ratio as a function of temperature for several of the cases $(\$ 4.2)$. These conditional means capture a qualitative shift in the burning as the flame becomes distributed. We also use a horizontal averaging approach to look at average profiles (\$4.3.2), scalar conservation balance $(\$ 4.3 .3)$ and the scalar fluctuation transport equation (\$4.4.1). Finally, the simulations are discussed in $\S 5$ with an emphasis on the characteristics of distributed flames.

\section{Numerical approach}

The numerical approach taken here is based on a low-Mach-number formulation of the reacting Navier-Stokes equations. The low-Mach-number model, derived from 
asymptotic analysis (see Rehm \& Baum 1978; Majda \& Sethian 1985) analytically filters sound waves from the system and is valid when flow velocities in the system are small with respect to the sound speed, as expected here. A mixture-averaged approximation for differential/preferential diffusion is used that neglects the Soret and Dufour transport (see Ern \& Giovangigli 1994). In the absence of gravity and radiative transport processes, the resulting equations of motion are

$$
\begin{aligned}
\frac{\partial \rho}{\partial t}+\nabla \cdot(\rho \boldsymbol{u}) & =0, \\
\frac{\partial}{\partial t}(\rho \boldsymbol{u})+\nabla \cdot(\rho \boldsymbol{u} \boldsymbol{u}) & =-\nabla \pi+\nabla \cdot \boldsymbol{\tau}+\rho \boldsymbol{F}, \\
\frac{\partial}{\partial t}\left(\rho Y_{i}\right)+\nabla \cdot\left(\rho Y_{i} \boldsymbol{u}\right) & =\nabla \cdot\left(\rho D_{i} \nabla Y_{i}\right)+\rho \omega_{i}, \\
\frac{\partial}{\partial t}(\rho h)+\nabla \cdot(\rho h \boldsymbol{u}) & =\nabla \cdot(\lambda \nabla T)+\sum_{i} \nabla \cdot\left(\rho h_{i} D_{i} \nabla Y_{i}\right),
\end{aligned}
$$

where $\rho$ is the density, $\boldsymbol{u}$ is the velocity, $Y_{i}$ is the mass fraction of species $i, h$ is the mixture enthalpy, $T$ is the temperature, $\rho \omega_{i}$ is the net mass production rate for species $i$ due to chemical reactions, $\boldsymbol{F}$ is a long-wavelength forcing term to drive turbulent fluctuation (discussed in more detail below), $\lambda$ is the thermal conductivity, $\boldsymbol{\tau}$ is the stress tensor, and $h_{i}(T)$ and $D_{i}$ are the enthalpy and species' mixture-averaged diffusion coefficients of species $i$, respectively. In the low-Mach-number formulation, the perturbational pressure field, $\pi$, satisfies $\pi / p_{0} \sim O\left(M a^{2}\right)$, where $p_{0}$ is the ambient thermodynamic pressure and $M a$ is the Mach number. Here, $p_{0}=1 \mathrm{~atm}$ and remains constant in time and space to represent an 'open' system. The fluid is treated as a mixture of perfect gases with an ideal equation of state written as

$$
p_{0}=\rho R_{\text {mix }} T=\rho \mathscr{R} T \sum_{i} \frac{Y_{i}}{W_{i}},
$$

where $W_{i}$ is the molecular weight of species $i$, and $\mathscr{R}$ is the universal gas constant. The transport coefficients, thermodynamic relationships and hydrogen kinetics are specified via the GRIMech 2.11 model (Bowman et al. 1995), with the relevant carbon species removed. The model includes nine chemical species and 27 fundamental Arrhenius reactions, and incorporates the molecular transport processes known to play a significant role in the dynamics of cellular flames. Although the current study is focused on flame propagation effects, use of this detailed model avoids unnecessary simplifications (such as single, fixed Lewis number or single-step global reactions), which might significantly affect salient aspects of the turbulence-flame interaction.

The basic discretisation (Day \& Bell 2000) combines a symmetric operator-split treatment of chemistry and transport with a high-resolution fractional step approach for advection. A density-weighted approximate projection (Almgren, Bell \& Szymczak 1996; Almgren, Bell \& Crutchfield 2000) ensures that the evolution of (2.1)-(2.4) satisfies the constraint imposed by the equation of state (2.5), see Pember et al. (1995). The low-Mach-number formulation eliminates the acoustic wave propagation from the system, enabling numerical evolution at the time scales of advective transport, resulting in an order-of-magnitude gain in overall integration efficiency. Diffusion and chemical kinetics, which occur on time scales faster than advection, are treated time-implicitly. This integration scheme is embedded in a parallel adaptive projection framework based on a hierarchical system of rectangular grid patches (Almgren et al. 


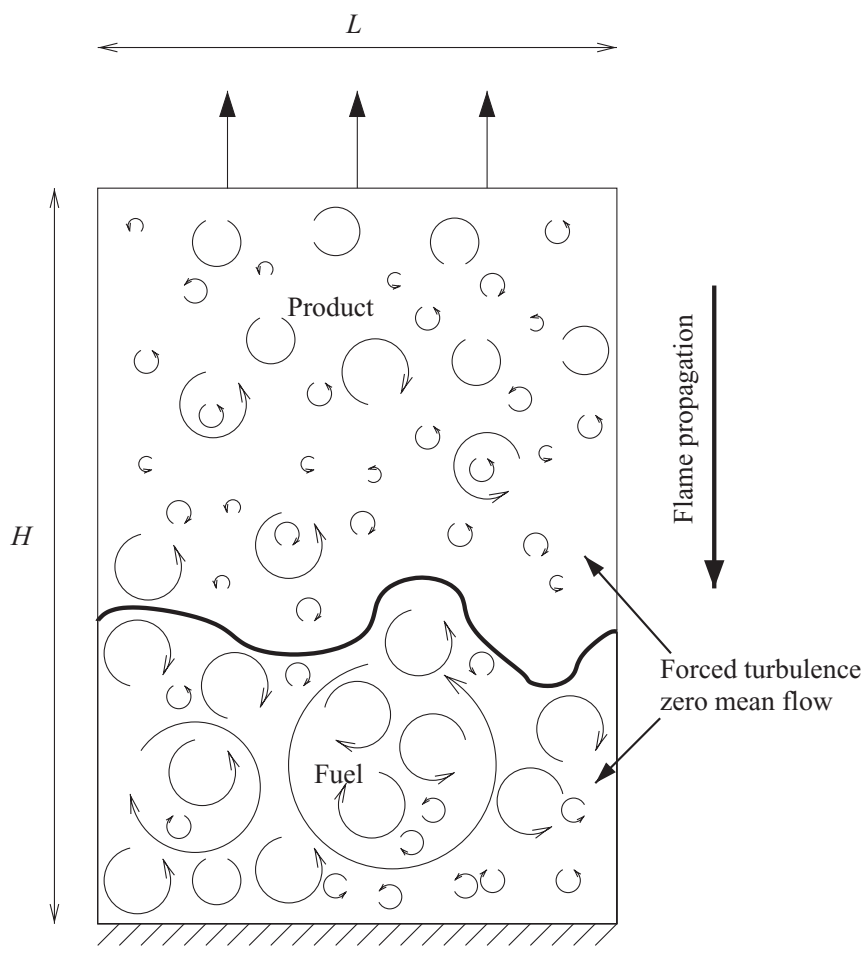

FIGURE 3. Schematic diagram of the flow configuration. A turbulent background was maintained in a high-aspect-ratio domain using a superposition of long-wavelength Fourier modes. Lateral periodic boundary conditions were used with a solid base and outflow at the top. The flame was initialised with fuel beneath products resulting in a downward-propagating flame.

2000). The overall adaptive integration algorithm is second-order accurate in space and time, and discretely conserves species mass and enthalpy.

The overall numerical scheme has been demonstrated to converge with secondorder accuracy and has been extensively validated in a variety of configurations. Two-dimensional laminar flames including vortex-flame interaction were studied in Bell et al. (2000) and emissions from laminar diffusion flames in Bell et al. (2002b) and Sullivan et al. (2002). The methodology has also been used for three-dimensional DNS of a turbulent premixed flame with detailed chemistry for methane (Bell, Day \& Grcar 2002a) and hydrogen (Day et al. 2009a). In addition, the methodology has been used to simulate laboratory-scale turbulent premixed flames, including a rodstabilised V-flame (Bell et al. 2005), a piloted slot Bunsen burner (Bell et al. 2007b) and a low swirl burner (Bell et al. 2008; Day et al. 2009b).

\section{Problem definition}

The approach used here follows Aspden et al. (2008a), where a similar study was conducted in type Ia supernovae. Figure 3 shows a schematic diagram of the flow configuration. The flow was initialised with cold fuel beneath hot products, resulting in a downward-propagating flame. Periodic lateral boundary conditions were specified, along with an insulating free-slip fixed wall at the bottom of the domain and outflow at the top. A high-aspect-ratio domain $(1: 1: 8)$ was used to give the flame sufficient space 
through which to propagate and enable the collection of ample statistics for analysis. A forcing term was used in the momentum equations to maintain the turbulent background. This approach is preferred to using an inflow boundary as it allows the turbulence to be maintained rather than decay, avoids detrimental Rayleigh-Taylor instabilities introduced by a variable inflow rate that would be necessary to account for the changing turbulent flame speed, both of which are exaggerated in low-Lewisnumber flames, but most importantly allows arbitrarily large turbulence levels that are numerically incompatible with an inflow boundary condition. The same densityweighted forcing term in Aspden et al. (2008b) and the supernova study (Aspden et al. 2008a) was used. An initial turbulent velocity field was obtained by running an inert calculation using the same forcing term until the turbulence was well-developed. A one-dimensional unstretched laminar flame was computed in an auxiliary step using the PREMIX code (Kee et al. 1983) incorporating identical chemical, transport and thermodynamics models. The resulting flame profiles were superimposed over the turbulent velocity. The final step in the initialisation was to project this initial velocity field using the above methodology to incorporate the expansion across the flame. The forcing term was used to maintain the turbulence during the reacting part of the simulation. In each case, the flame evolves through an initial transient until a turbulent flame is established that is statistically steady in the reference frame moving with the flame, to be discussed in detail below. This numerical configuration does not have a direct experimental analogue, but is designed to examine turbulenceflame interactions on length scales comparable with the flame thickness and uses the momentum forcing term to mimic the turbulent cascade produced by larger scales not present in the simulation.

There are two demands on computational resolution in this configuration. First, the structure of the flame needs to be resolved. In all of the simulations presented here, the flames are resolved with a grid spacing $\Delta x<l_{L} / 25$, where $l_{L}$ is the thermal thickness of the corresponding PREMIX solution. This was found to be more than sufficient to resolve the thermal and chemical structure of the flame and to recover the value of $s_{L}$ computed with the corresponding resolved PREMIX solution. Second, the smallest scales in the turbulent cascade must be resolved. The ability of the core discretisation scheme to perform well-resolved simulations of turbulent flow simulations was examined in Aspden et al. (2008b), where an empirical characterisation through an effective viscosity was presented. In each simulation, the Kolmogorov length scale has been estimated as $\eta=\left(v^{3} / \varepsilon\right)^{1 / 4}$, with $\varepsilon=\breve{u}^{3} / l$ where $\breve{u}$ and $l$ are the turbulent intensity and integral length scale, respectively. The effective Kolmogorov length scale $\left(\eta_{e}\right)$ of the simulation is calculated according to the formula derived in Aspden et al. (2008b). The resolution was found to be sufficient when the condition $\eta_{e}<1.5 \eta$ was satisfied, and both quantities are given in the tables describing the respective simulations. Further details and convergence studies for several pertinent cases are presented in the Appendix.

Adaptive mesh refinement (AMR) was used to focus resolution around the flame, reducing computational expense elsewhere in the domain. In each case, the simulation was run without refinement until the steady state is reached, thus establishing the flame at reduced computational expense, then the refinement was added so that useful data could be collected at the highest resolution after a brief period of equilibration. A buffer region was used ahead of the flame to allow the turbulence to spin-up, i.e. to guarantee sufficient time for the turbulent cascade to populate the finer scales. The size of the buffer was determined by running inert homogeneous isotropic turbulence and looking at the kinetic energy wavenumber spectra. The high-wavenumber modes 
were found to become fully populated within 10-15 time steps. Therefore, a 16-cell buffer was used as this provides a conservative bound on the distance information can travel in 16 time steps bounded by the Courant-Friedrichs-Lewy (CFL) condition.

The one-dimensional unstretched laminar flame speeds and thicknesses (computed with PREMIX) were used to define the domain size and turbulent intensities in each simulation. The domain width was the same in all cases, $L=5 l_{L}$, and velocity ratios $\check{u} / s_{L}$ of approximately 3.7, 17, 33 and 107 were used, giving Karlovitz numbers of 10, 100, 266 and 1562, and Damköhler numbers ranging from approximately 0.14 to $4.7 \times 10^{-3}$. The required turbulent intensities were obtained by varying the amplitudes of the forcing term. Previous work has shown that this forcing term gives an integral length scale, defined as the integral of the longitudinal correlation function, of $l \approx L / 10$, and so $l_{L}=2 l$. This is satisfactory because we are primarily interested in disrupting the internal structure of the flame.

\section{Results}

\subsection{Phenomenology of flame response to turbulence}

\subsubsection{Cellular burning}

The cellular burning patterns that form naturally in lean hydrogen-air mixtures are most readily apparent in the low-turbulence cases. Figure 4 shows a perspective view of the $Y_{\mathrm{H}_{2}}=0.001$ contour, extracted from a snapshot of a $\varphi=0.31$ case at $K a_{L}=10$ after a statistically steady flame has been established (referred to as A31). For orientation, the computational domain is outlined with thin black lines, and the view is taken from outside the box at a small distance below the flame. The contour surface is dominated by fairly large-scale structures that are extremely convoluted. The contour is coloured by the local value of $Y_{\mathrm{OH}}$. In lean hydrogen-air flames, the $\mathrm{OH}$ concentration correlates robustly with the fuel consumption (see, for example, Bell et al. 2007a) and is frequently used in laboratory experiments as a marker of the flame position. The $\mathrm{OH}$ concentrations in the figure show considerable variation over the surface, with local values ranging from zero to as much as 100 times the value observed in the corresponding PREMIX solution at $Y_{\mathrm{OH}}=0.001$. The most intensely burning regions appear to occur in regions of high curvature where the contour bulges towards the cold fuel. Although not apparent in the figure, high values of $\mathrm{OH}$ occur in small lens-shaped volumes that align roughly with the fuel contour shown. These cellular structures correspond to regions of superadiabatic gas temperature and elevated fuel consumption.

\subsubsection{Effect of Karlovitz number at $\varphi=0.31$}

Table 1 summarises the parameters for four simulations at $\varphi=0.31$, referred to as cases A31-D31 (with increasing Karlovitz number). As the domain width has been set according to the unstretched laminar flame thickness, all of these simulations have the same domain size. Note that case C31 has a Karlovitz number, $K a=266$, which is similar to the distributed flame in the supernova study of Aspden et al. (2008a) $(K a=230)$, while in case D31 $(K a=1562) \check{u} / s_{L}>100$. Also note the comparison of the effective Kolmogorov length scales $\eta_{e}$ with the Kolmogorov length scale estimated from the turbulence properties (see the convergence study in the Appendix for case C31).

Figure 5 presents vertical slices through the domain for the four simulations, taken from snapshots of each solution after the turbulent flames have become established. We note that the choice of temporal and spatial positions of the slices is arbitrary 


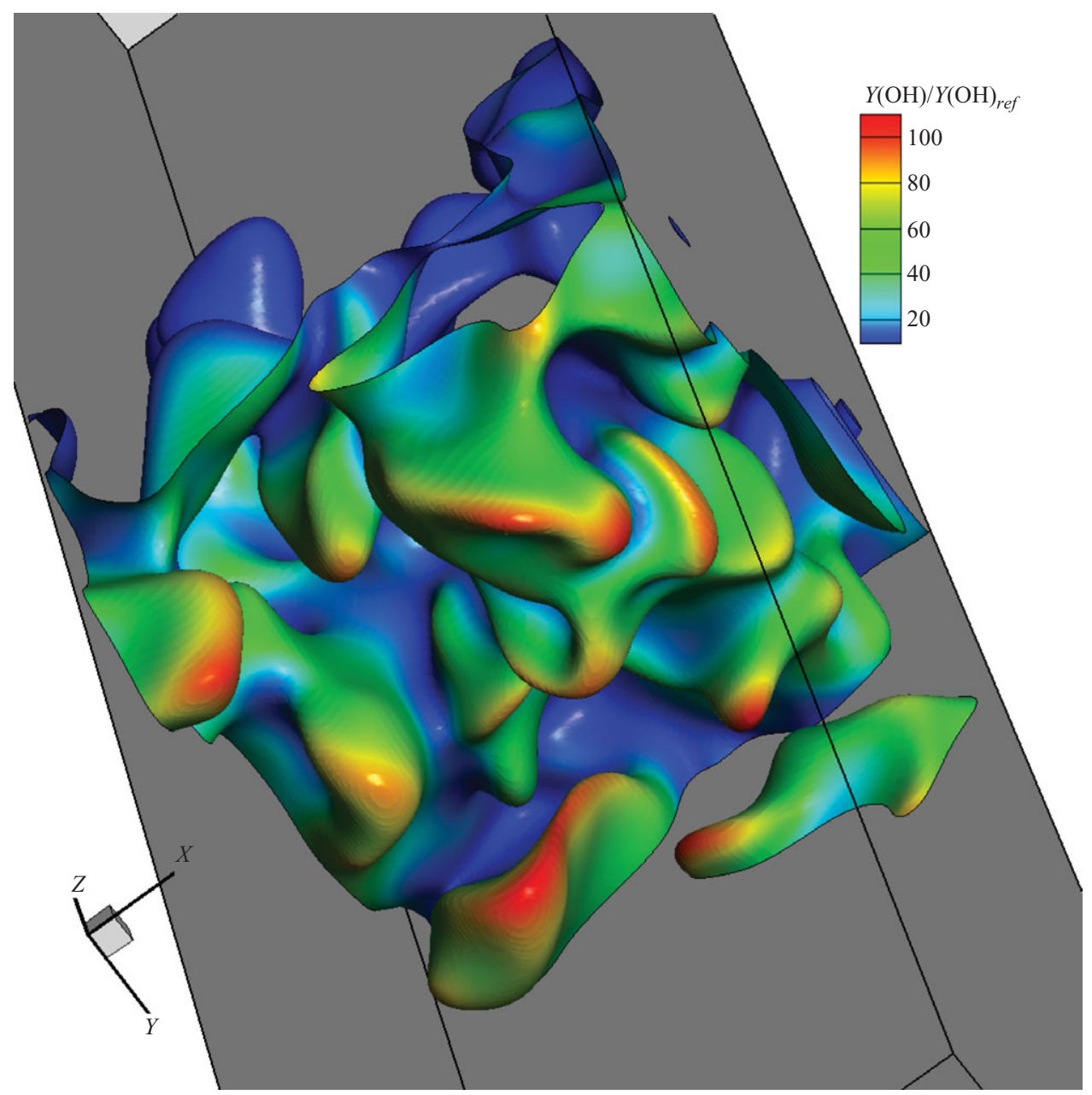

FIGURE 4. Contour of $Y_{\mathrm{H}_{2}}=0.001$ extracted from a snapshot of the low-turbulence case A31. Contour is coloured by the local value of $Y_{\mathrm{H}_{2}}$, normalised by the corresponding PREMIX value at the same value of $Y_{\mathrm{H}_{2}}$.

because the flow is statistically stationary and the lateral boundary conditions are periodic; the slices shown are representative of each flame. The quantities plotted are density, $\mathrm{H}_{2}$ consumption rate and temperature. Note that only three-quarters of the domain height is shown. In cases A31 and B31, there is strong burning in regions of positive curvature (where the centre of curvature is on the products side of the flame) separated by local extinction regions typical of the cellular burning structure in thermodiffusively unstable lean hydrogen flames, as seen in figure 4. In these regions, preferential diffusion leads to local enrichment of the fuel, and the flame burns at a locally higher equivalence ratio (see Day et al. 2009a).

As the Karlovitz number is increased, this cellular burning structure is increasingly disrupted. The turbulence is able to move the flame around and wrinkle the surface, creating a greater flame surface area. There is a decrease in the individual structure size, which gives rise to higher curvatures, greater focusing of hydrogen and higher local burning rates. The vertical extent over which there is significant fuel consumption 
Case

Equivalence ratio $(\varphi)$

Flame speed $\left(s_{L}\right)\left(\mathrm{m} \mathrm{s}^{-1}\right)$

Flame width $\left(l_{L}\right)(\mathrm{m})$

Domain width $(L)(\mathrm{m})$

Domain height $(H)(\mathrm{m})$

Integral length scale $(l)(\mathrm{m})$

Length ratio $\left(l / l_{L}\right)$

RMS velocity $(\check{u})\left(\mathrm{m} \mathrm{s}^{-1}\right)$

Velocity ratio $\left(\breve{u} / s_{L}\right)$

Karlovitz number $\left(K a_{L}\right)$

Damköhler number $\left(D a_{L}\right)$

Levels of refinement

Effective resolution $(N)$

Cell width $(\Delta x)(\mathrm{m})$

Kolmogorov length $(\eta)(\mathrm{m})$

Cell Kolmogorov length $\left(\eta_{\Delta x}\right)(\mathrm{m})$

Effective Kolmogorov length $\left(\eta_{e}\right)(\mathrm{m})$

$\begin{array}{cccc}\mathrm{A} 31 & \mathrm{~B} 31 & \mathrm{C} 31 & \mathrm{D} 31 \\ 0.31 & 0.31 & 0.31 & 0.31 \\ 4.68 \times 10^{-2} & 4.68 \times 10^{-2} & 4.68 \times 10^{-2} & 4.68 \times 10^{-2} \\ 1.9 \times 10^{-3} & 1.9 \times 10^{-3} & 1.9 \times 10^{-3} & 1.9 \times 10^{-3} \\ 9.5 \times 10^{-3} & 9.5 \times 10^{-3} & 9.5 \times 10^{-3} & 9.5 \times 10^{-3} \\ 7.6 \times 10^{-2} & 7.6 \times 10^{-2} & 7.6 \times 10^{-2} & 7.6 \times 10^{-2} \\ 9.5 \times 10^{-4} & 9.5 \times 10^{-4} & 9.5 \times 10^{-4} & 9.5 \times 10^{-4} \\ 0.5 & 0.5 & 0.5 & 0.5 \\ 0.173 & 0.8 & 1.54 & 5.0 \\ 3.69 & 17.1 & 32.9 & 106.8 \\ 10 & 100 & 266 & 1562 \\ 1.36 \times 10^{-1} & 2.92 \times 10^{-2} & 1.52 \times 10^{-2} & 4.68 \times 10^{-3} \\ 1 & 1 & 1 & 2 \\ 128^{2} \times 1024 & 128^{2} \times 1024 & 128^{2} \times 1024 & 256^{2} \times 2048 \\ 7.42 \times 10^{-5} & 7.42 \times 10^{-5} & 7.42 \times 10^{-5} & 3.711 \times 10^{-5} \\ 1.85 \times 10^{-4} & 5.85 \times 10^{-5} & 3.58 \times 10^{-5} & 1.48 \times 10^{-5} \\ 2.23 \times 10^{-5} & 2.23 \times 10^{-5} & 2.23 \times 10^{-5} & 1.11 \times 10^{-5} \\ 1.85 \times 10^{-4} & 6.05 \times 10^{-5} & 4.13 \times 10^{-5} & 1.83 \times 10^{-5}\end{array}$

TABLE 1. Turbulent flame properties for the four simulations at equivalence ratio $\varphi=0.31$.

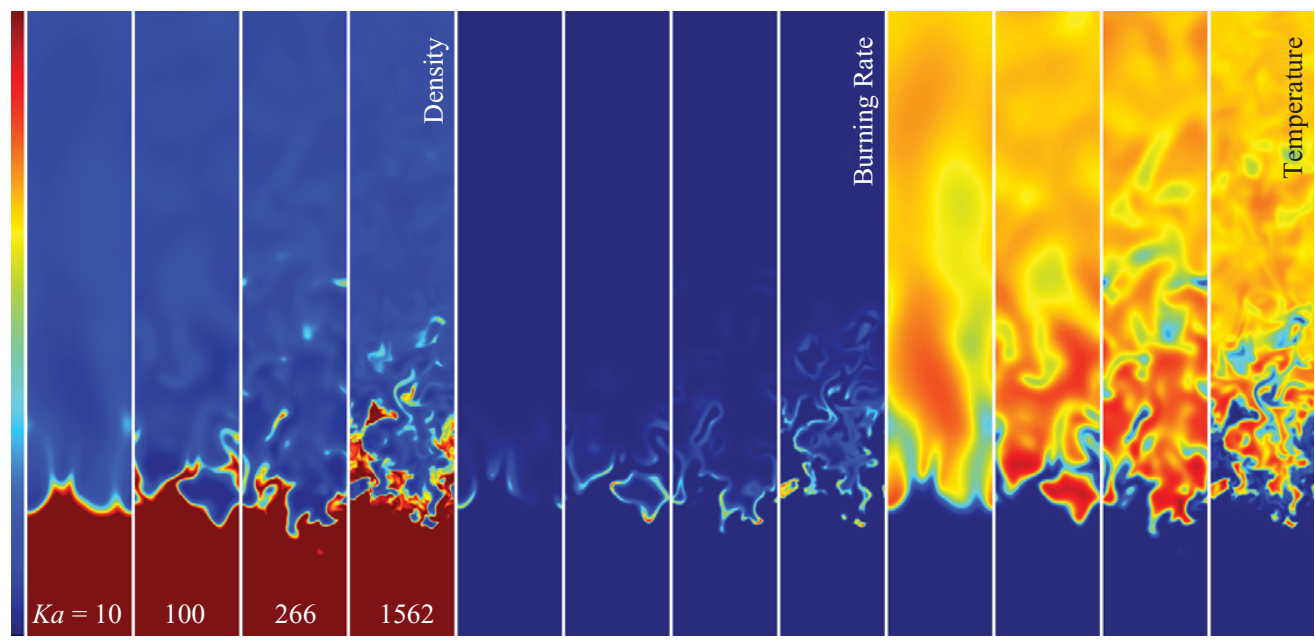

FIGURE 5. Two-dimensional vertical slices through three-dimensional simulations showing density, burning rate and temperature at $\varphi=0.31$, respectively. The density range is $[0.2,1.02] \mathrm{kg} \mathrm{m}^{-3}$ in each case. The burning rate is shown between zero and $15,25,35$ and $45 \mathrm{~kg} \mathrm{~m}^{-3} \mathrm{~s}^{-1}$, respectively. The temperature range is $[298,1600] \mathrm{K}$ in each case.

becomes much broader. In all cases, there is a sharp interface between the fuel and the products. Even at a Karlovitz number of 1562, a distributed flame is not observed (to be discussed in more detail below). The turbulence is able to draw volumes of pure fuel into the products before they can burn, but is not able to disrupt the internal structure of the flame. 


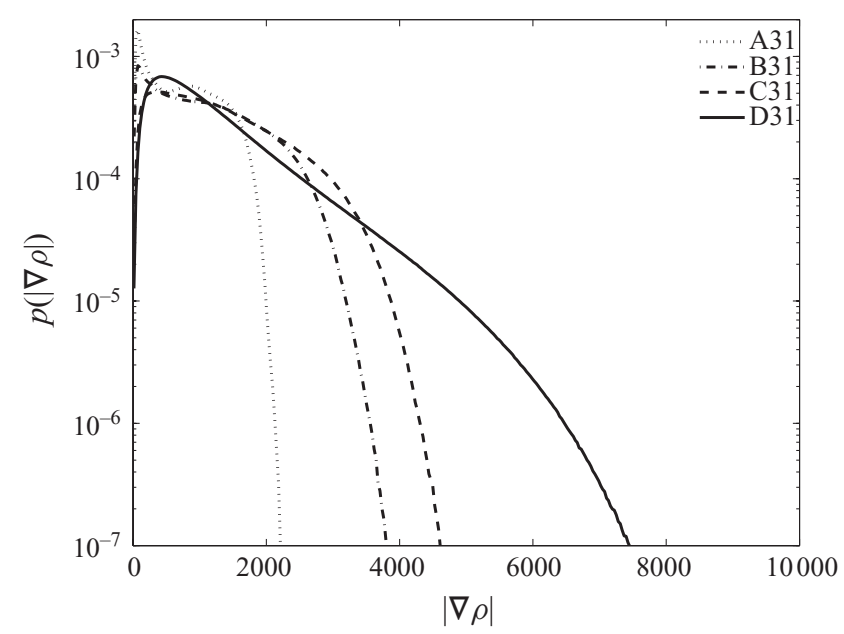

FIGURE 6. Probability distribution functions of $|\nabla \rho|$ for cases A31-D31.

The change in the individual structures can be highlighted by considering the PDF of the density gradients. Figure 6 shows the PDF of $|\nabla \rho|$ for cases A31-D31, plotted semilogarithmically. To remove the large regions of pure fuel and pure products, the data have been conditioned on density being between the $10 \%$ and $90 \%$ thresholds of the PREMIX solution. As the Karlovitz number increases, the PDF contains regions with higher gradients, implying that there are regions with much sharper interfaces than in the lower Karlovitz number flames. This is consistent with the observation that increased turbulence leads to more wrinkling of the flame surface, which leads to increased focusing by hydrogen diffusion, enhancing the local burning rate and sharpening the interface between fuel and product.

Figure 7 shows the turbulent flame speeds for the four cases as a function of time (normalised by the eddy turnover time of the integral length scale $\tau_{T}=l / \check{u}$ ). The turbulent flame speed is defined here as the rate of change of fuel mass fraction integrated over the domain divided by the product of the cross-sectional area of the domain and the mass fraction of the pure fuel,

$$
s_{T}(t) \equiv \frac{1}{L^{2}\left(\rho Y_{\left.\mathrm{H}_{2}\right)_{0}}\right.} \frac{\mathrm{d}}{\mathrm{d} t} \int_{V} \rho Y_{\mathrm{H}_{2}} \mathrm{~d} V .
$$

The mean and standard deviation in cases A31-D31 are $0.42 \pm 0.055,1.52 \pm 0.20$, $2.90 \pm 0.50$ and $4.54 \pm 0.82 \mathrm{~m} \mathrm{~s}^{-1}$, respectively. In each case, the dotted line denotes the low-resolution part of the simulation used to establish the flame, and the solid lines denote continuations of each simulation with increased grid refinement focused in a broad layer near the flame front. The flat line denotes the average flame speed and the temporal range $\left[t_{0}, t_{1}\right]$ over which the averaging was taken. It is clear that there is a large variation in the mean flame speed in all cases; these flames are unstable and the turbulence compounds this effect. Note that the simulations have been run for a significant number of integral length eddy turnover times.

As the Karlovitz number is increased, the turbulent flame speed also increases. Figure $7(b)$ suggests that the data are well approximated by a power law at lowto-moderate Karlovitz numbers (the exponent is approximately 0.59). At the highest Karlovitz number, the turbulent flame speed is slower than predicted by the power 
Case

Equivalence ratio $(\varphi)$

Flame speed $\left(s_{L}\right)\left(\mathrm{m} \mathrm{s}^{-1}\right)$

Flame width $\left(l_{L}\right)(\mathrm{m})$

Domain width $(L)(\mathrm{m})$

Domain height $(H)(\mathrm{m})$

Integral length scale $(l)(\mathrm{m})$

Length ratio $\left(l / l_{L}\right)$

RMS velocity $(\check{u})\left(\mathrm{m} \mathrm{s}^{-1}\right)$

Velocity ratio $\left(\breve{u} / s_{L}\right)$

Karlovitz number $\left(K a_{L}\right)$

Damköhler number $\left(D a_{L}\right)$

Levels of refinement

Effective resolution $(N)$

Cell width $(\Delta x)(\mathrm{m})$

Kolmogorov length $(\eta)(\mathrm{m})$

Cell Kolmogorov length $\left(\eta_{\Delta x}\right)(\mathrm{m})$

Effective Kolmogorov length $\left(\eta_{e}\right)(\mathrm{m})$

$\begin{array}{cccc}\text { A } 40 & \mathrm{~B} 40 & \mathrm{C} 40 & \mathrm{D} 40 \\ 0.40 & 0.40 & 0.40 & 0.40 \\ 2.24 \times 10^{-1} & 2.24 \times 10^{-1} & 2.24 \times 10^{-1} & 2.24 \times 10^{-1} \\ 6.29 \times 10^{-4} & 6.29 \times 10^{-4} & 6.29 \times 10^{-4} & 6.29 \times 10^{-4} \\ 3.14 \times 10^{-3} & 3.14 \times 10^{-3} & 3.14 \times 10^{-3} & 3.14 \times 10^{-3} \\ 2.512 \times 10^{-2} & 2.512 \times 10^{-2} & 2.512 \times 10^{-2} & 2.512 \times 10^{-2} \\ 3.14 \times 10^{-4} & 3.14 \times 10^{-4} & 3.14 \times 10^{-4} & 3.14 \times 10^{-4} \\ 0.5 & 0.5 & 0.5 & 0.5 \\ 0.825 & 3.83 & 7.34 & 23.9 \\ 3.69 & 17.1 & 32.9 & 106.8 \\ 10 & 100 & 266 & 1562 \\ 1.36 \times 10^{-1} & 2.92 \times 10^{-2} & 1.52 \times 10^{-2} & 4.68 \times 10^{-3} \\ 1 & 1 & 1 & 2 \\ 128^{2} \times 1024 & 128^{2} \times 1024 & 128^{2} \times 1024 & 256^{2} \times 2048 \\ 2.45 \times 10^{-5} & 2.45 \times 10^{-5} & 2.45 \times 10^{-5} & 1.23 \times 10^{-5} \\ 4.33 \times 10^{-5} & 1.37 \times 10^{-5} & 8.41 \times 10^{-6} & 3.47 \times 10^{-6} \\ 7.36 \times 10^{-6} & 7.36 \times 10^{-6} & 7.36 \times 10^{-6} & 3.68 \times 10^{-6} \\ 4.33 \times 10^{-5} & 1.51 \times 10^{-5} & 11.2 \times 10^{-6} & 5.12 \times 10^{-6}\end{array}$

TABLE 2. Turbulent flame properties for the four simulations at equivalence ratio $\varphi=0.40$.

(a)

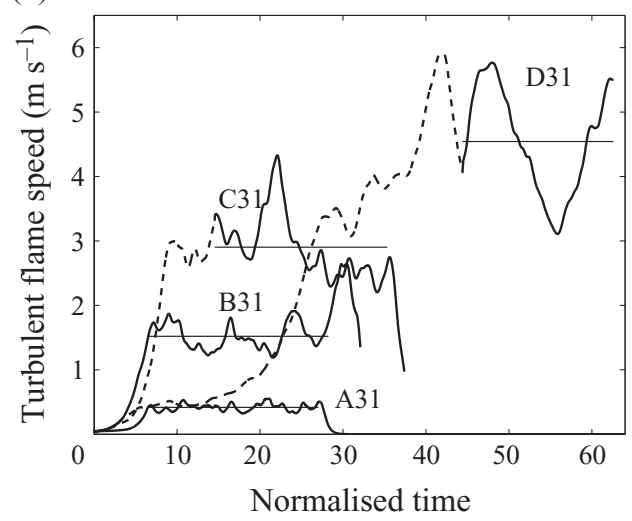

(b)

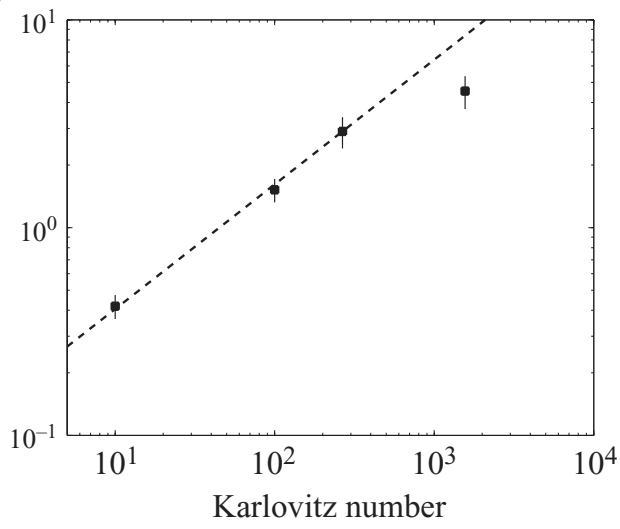

FIGURE 7. Turbulent flame speeds for cases A31-D31 against (a) time normalised by integral length turnover time and $(b)$ Karlovitz number.

law. However, at approximately $5 \mathrm{~m} \mathrm{~s}^{-1}$, the turbulent flame speed is approximately 100 times greater than $s_{L}$.

\subsubsection{Effect of Karlovitz number at $\varphi=0.40$}

In this section, we present four simulations over the same range of Karlovitz numbers as those presented above at a higher equivalence ratio, $\varphi=0.40$. Table 2 summarises the simulation properties, referred to as A40-D40. Since the richer flame is thinner and faster, the domain size is smaller and the turbulent intensities are higher in cases A40-D40 than A31-D31. 


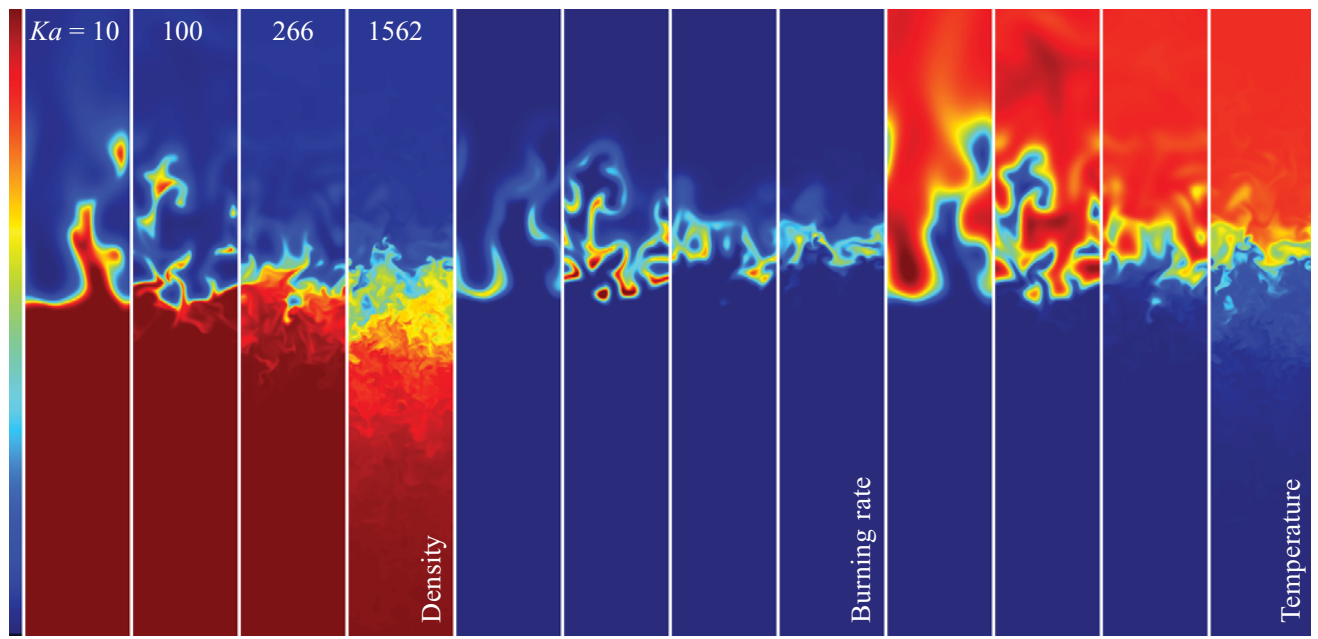

FIgURE 8. Two-dimensional vertical slices through three-dimensional simulations showing density, burning rate and temperature at $\varphi=0.40$, respectively. The density, burning rate and temperature ranges are $[0.2,1.02] \mathrm{kg} \mathrm{m}^{-3},[0,64] \mathrm{kg} \mathrm{m}^{-3} \mathrm{~s}^{-1}$ and $[298,1600] \mathrm{K}$, respectively.

Figure 8 shows slices through the domain that are analogous to those in figure 5 . Again, only three-quarters of the domain height is shown. A similar response is seen for cases A40 and B40 as in $\S 4$.1.2. Specifically, the turbulence reduces the individual structure size and intensifies the local burning rate, and there is a slight increase in the vertical extent over which there is significant fuel consumption. However, a different trend is observed for cases B40-D40. The cellular burning structure is increasingly disrupted, and the vertical extent of the burning is reduced. Visually, the density field for case D40 resembles a turbulent mixing zone (e.g. the Rayleigh-Taylor instability). There is no longer a sharp interface between the fuel and the products. The local burning rate has decreased, although it is still higher than in the unstretched laminar flame, with burning occurring in a narrow band close to the high-temperature end of the distribution. These features are similar to those observed in the distributed flame in the supernova study (Aspden et al. 2008b); however, the present flame does not appear to be distributed to the same extent (see Aspden et al. 2008a and figure 2). Case C40 appears to be transitional.

An interesting feature of case D40 is that there are no longer hot spots in the flow, where we consider a hot spot to be a region that exceeds the adiabatic flame temperature. In cases A40 and B40, the peak temperature is approximately $1700 \mathrm{~K}$, whereas in case D40, the peak temperature is approximately $1420 \mathrm{~K}$, a reduction of almost $300 \mathrm{~K}$. This reduction in peak temperature could have potential significance for the formation of thermal $\mathrm{NO}_{x}$ emissions. We note that the absence of hot spots is a consequence of the transition to distributed burning, but will only manifest in low-Lewis-number flames.

Figure $9(a)$ shows the PDF of $|\nabla \rho|$ for cases A40-D40. Again, the data have been conditioned on the $10 \%$ and $90 \%$ thresholds of the corresponding one-dimensional unstretched laminar flame. At low-to-moderate Karlovitz numbers, a similar trend is observed as in the $\varphi=0.31$ cases; higher gradients are observed at higher Karlovitz numbers. However, for case D40, the trend is reversed; much lower gradients are observed, which highlights a significant change in the interface between the fuel 

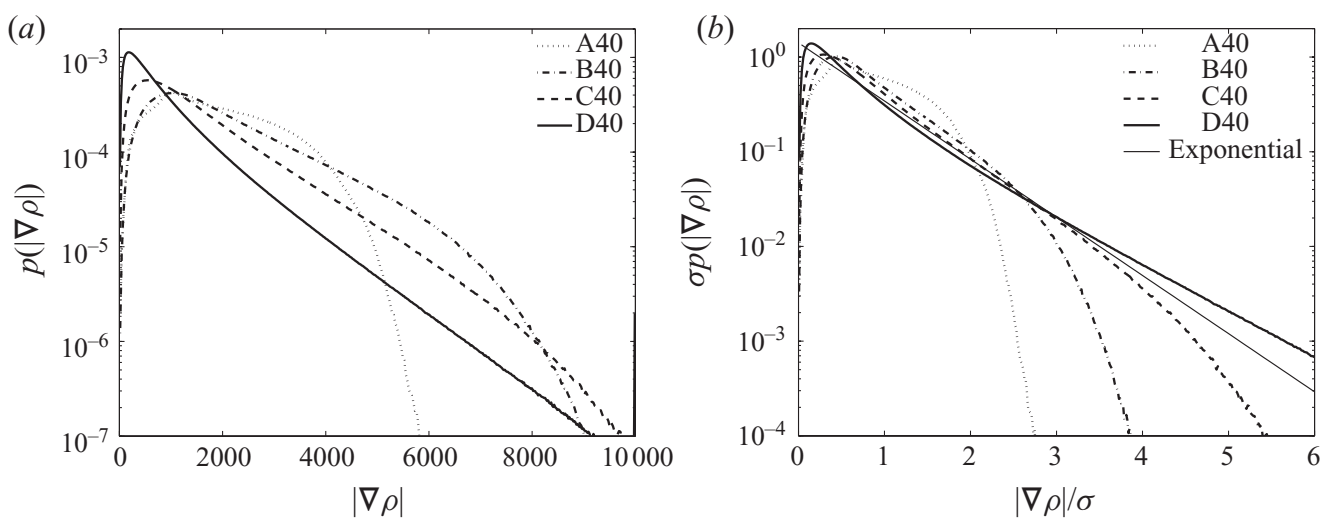

FIgURE 9. Probability distribution functions of $|\nabla \rho|$ for cases A40-D40. Plot $(b)$ has been normalised by the standard deviation and plot $(a)$ has not.

and products. The sharp interface observed in cases A31-D31 and A40-C40 is not observed in case D40. Figure $9(b)$ shows the same PDFs, but normalised by the standard deviation $\sigma$. As the Karlovitz number increases, there is a clear trend towards an exponential distribution (shown by the thin straight line). The kurtoses of the four cases are $K=2.0,3.0,4.5$ and 7.7, respectively ( $K=3$ and $K=6$ for Gaussian and exponential distributions, respectively). Even though the kurtosis is sensitive to the arbitrary thresholds $(10 \%-90 \%)$ used to condition the data, the trend is robust and the PDFs are largely unaffected. PDFs with exponential tails have been observed in many examples of high-Reynolds-number scalar mixing, see Gollub et al. (1991), Jayesh \& Warhaft (1991) and Thoroddsen \& Van Atta (1992) as examples, and are indicative of the dominant role of turbulent mixing in case D40. This is also consistent with the observations of Dunn et al. (2009).

The turbulent flame speeds for cases A40-D40 are $1.36 \pm 0.25,3.58 \pm 0.96$, $3.44 \pm 1.02$ and $2.92 \pm 0.35 \mathrm{~m} \mathrm{~s}^{-1}$, respectively. At low-Ka, the turbulent flame speeds display an increasing trend. However, at this equivalence ratio, the turbulent flame speed rolls over at a Karlovitz number below 266 and is followed by a decreasing trend. Here, the fastest turbulent flame speed observed is just over $20 s_{L}$. In case D40, the variation of the turbulent flame speed around the mean appears to be much lower than in the other cases; the distributed hydrogen flame burns at a more uniform speed.

\subsubsection{Effect of equivalence ratio at $K a_{L}=266$}

Despite the fact that the two previous sections compared flames at the same Karlovitz and Damköhler numbers, clear differences were found. To explore this further, two additional simulations were run at $K a_{L}=266$, but with different equivalence ratios, specifically cases C34 and C37. Table 3 summarises the simulation properties. Note that with increasing equivalence ratio, the unstretched laminar flame speed increases and the width decreases; therefore, to achieve the same $K a_{L}$ and $D a_{L}$, the physical domain size of these simulations decreases with equivalence ratio and the absolute turbulent intensity increases.

Figure 10 shows the slices through the domain as before. The variability in temperature in the post-flame region decreases with increasing equivalence ratio, which is indicative of the decreasing degree of enhancement in the burning intensity resulting 


\begin{tabular}{lcccc}
\hline & & & & \\
Case & $\mathrm{C} 31$ & $\mathrm{C} 34$ & $\mathrm{C} 37$ & $\mathrm{C} 40$ \\
Equivalence ratio $(\varphi)$ & 0.31 & 0.34 & 0.37 & 0.40 \\
Flame speed $\left(s_{L}\right)\left(\mathrm{m} \mathrm{s}^{-1}\right)$ & $4.68 \times 10^{-2}$ & $9.34 \times 10^{-2}$ & $1.52 \times 10^{-1}$ & $2.24 \times 10^{-1}$ \\
Flame width $\left(l_{L}\right)(\mathrm{m})$ & $1.9 \times 10^{-3}$ & $1.1 \times 10^{-3}$ & $7.9 \times 10^{-4}$ & $6.29 \times 10^{-4}$ \\
Domain width $(L)(\mathrm{m})$ & $9.5 \times 10^{-3}$ & $5.5 \times 10^{-3}$ & $3.95 \times 10^{-3}$ & $3.14 \times 10^{-3}$ \\
Domain height $(H)(\mathrm{m})$ & $7.6 \times 10^{-2}$ & $4.4 \times 10^{-2}$ & $3.16 \times 10^{-2}$ & $2.512 \times 10^{-2}$ \\
Integral length scale $(l)(\mathrm{m})$ & $9.5 \times 10^{-4}$ & $5.5 \times 10^{-4}$ & $3.95 \times 10^{-4}$ & $3.14 \times 10^{-4}$ \\
Length ratio $\left(l / l_{L}\right)$ & 0.5 & 0.5 & 0.5 & 0.5 \\
RMS velocity $(\check{u})\left(\mathrm{m} \mathrm{s}^{-1}\right)$ & 1.54 & 3.07 & 5.0 & 7.34 \\
Velocity ratio $\left(\check{u} / s_{L}\right)$ & 32.9 & 32.9 & 32.9 & 32.9 \\
Karlovitz number $\left(K a_{L}\right)$ & 266 & 266 & 266 & 266 \\
Damköhler number $\left(D a_{L}\right)$ & $1.52 \times 10^{-2}$ & $1.52 \times 10^{-2}$ & $1.52 \times 10^{-2}$ & $1.52 \times 10^{-2}$ \\
Levels of refinement & 1 & 1 & & 1 \\
Effective resolution $(N)$ & $128^{2} \times 1024$ & $128^{2} \times 1024$ & $128^{2} \times 1024$ & $128^{2} \times 1024$ \\
Cell width $(\Delta x)(\mathrm{m})$ & $7.42 \times 10^{-5}$ & $4.30 \times 10^{-5}$ & $3.09 \times 10^{-5}$ & $2.45 \times 10^{-5}$ \\
Kolmogorov length $(\eta)(\mathrm{m})$ & $3.58 \times 10^{-5}$ & $1.87 \times 10^{-5}$ & $1.20 \times 10^{-5}$ & $8.41 \times 10^{-6}$ \\
Cell Kolmogorov length $\left(\eta_{\Delta x}\right)(\mathrm{m})$ & $2.23 \times 10^{-5}$ & $1.23 \times 10^{-5}$ & $9.23 \times 10^{-6}$ & $7.36 \times 10^{-5}$ \\
Effective Kolmogorov length $\left(\eta_{e}\right)(\mathrm{m})$ & $4.13 \times 10^{-5}$ & $2.23 \times 10^{-5}$ & $1.49 \times 10^{-5}$ & $11.2 \times 10^{-6}$
\end{tabular}

TABLE 3. Turbulent flame properties for the four simulations at Karlovitz number $K a_{L}=266$.

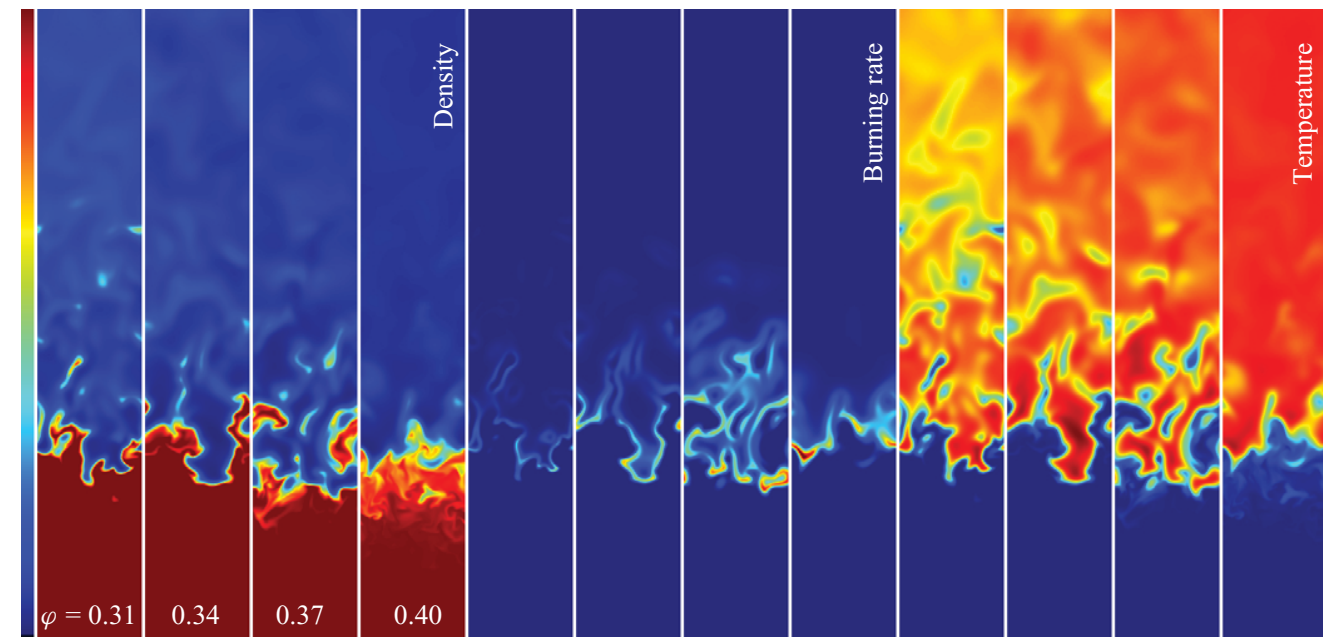

FIGURE 10. Two-dimensional vertical slices through three-dimensional simulations showing density, burning rate and temperature at $\varphi=0.31,0.34,0.34$ and 0.40 , respectively. The density, burning rate and temperature ranges are $[0.2,1.02] \mathrm{kg} \mathrm{m}^{-3},[0,64] \mathrm{kg} \mathrm{m}^{-3} \mathrm{~s}^{-1}$ and $[298,1600] \mathrm{K}$, respectively.

from the thermodiffusive instability as the flames become richer. For $\varphi=0.31-0.37$, the vertical extent where there is significant burning increases with equivalence ratio. For the richest case, this extent is significantly narrower, and the density mixing zone is broadened upstream of where burning occurs.

The turbulent flame speeds for the $\mathrm{C}$ cases do not vary significantly with equivalence ratio. The mean values are $2.90 \pm 0.50,3.97 \pm 0.76,3.60 \pm 0.79$ and $3.44 \pm 1.02 \mathrm{~m} \mathrm{~s}^{-1}$, 
respectively. There is an initial increase in turbulent flame speed, followed by a decreasing trend as equivalence ratio increases and the flame moves towards being distributed. This non-monotonic trend suggests that normalising the turbulent flame speed by turbulent intensity or $s_{L}$ will not provide much insight.

It appears that characterising premixed flames in terms of Karlovitz and Damköhler numbers is not universal for lean hydrogen. We suspect the reason for this is that the low-Lewis-number effects of lean hydrogen in more than one spatial dimension are not captured through the normalisation by $s_{L}$ and $l_{L}$. We will address this issue in a subsequent paper.

\subsection{Conditional means}

A useful way to examine the structure of turbulent flames, in particular the extent to which turbulent mixing is affecting the flame, is to look at the composition and temperature through the flame (see Aspden et al. 2008a and figure 2). Here we consider a similar diagnostic, but the data are presented through conditional means. Specifically, the mean and standard deviation of temperature were evaluated as a function of fuel mole fraction. Mole fraction was used as an independent variable to capture regions where the local temperature exceeds the adiabatic flame temperature. The integrals were averaged over a large number of time points after the flame had become statistically stationary.

Figure 11 shows the conditional mean of temperature as a function of $\mathrm{H}_{2}$ mole fraction for cases A31, A40, D31 and D40 (note that in this figure the independent variable (mole fraction) is shown on the ordinate). In each plot, the mean temperature is shown by the solid curve, and the distribution of the steady unstretched laminar flame is shown by the dashed curve. The standard deviation in temperature is shown by the shaded region. The flames burn from cold fuel at the top left in each figure, to the hot products at the bottom right (note the adiabatic flame temperature is denoted by the vertical dashed line). The unstretched laminar flame profiles lie below the linear path between fuel and products due to the low Lewis number of lean hydrogen. This is in contrast to the high-Lewis-number supernova distribution, which lies above the linear path (see Aspden et al. $2008 a$ and figure 2).

Cases A31 and A40 show broad distributions, close to the distributions of the corresponding unstretched laminar flame. As demonstrated by Day et al. (2009a), differential diffusion effects lead to a decorrelation of temperature and fuel, and results in regions that lie above the distribution of the unstretched laminar flame, i.e. the fuel is generally hotter than the corresponding point in the unstretched laminar flame. The hot spots are also evident here - temperatures are reached that exceed the adiabatic flame temperature. Case D40 has a distribution that is both narrow and far from the distribution of the unstretched laminar flame. This is consistent with the observations reported for the distributed supernova flame, which was attributed to the dominance of turbulent mixing. Case D31 appears to be transitional, i.e. there is competition between turbulent and molecular mixing processes. The distribution is mainly above the profile of the unstretched laminar flame, but the standard deviation remains high.

Unlike the supernovae, terrestrial combustion involves an oxidiser, which means there can be variations in the local equivalence ratio. Due to the comparatively large diffusivities, both atomic and molecular hydrogen diffuse downstream faster than the oxygen, giving rise to a lower equivalence ratio through the flame. Figure 12 shows conditional means for equivalence ratio as a function of temperature. Note here that it only makes sense to use temperature as the independent variable, and it is 

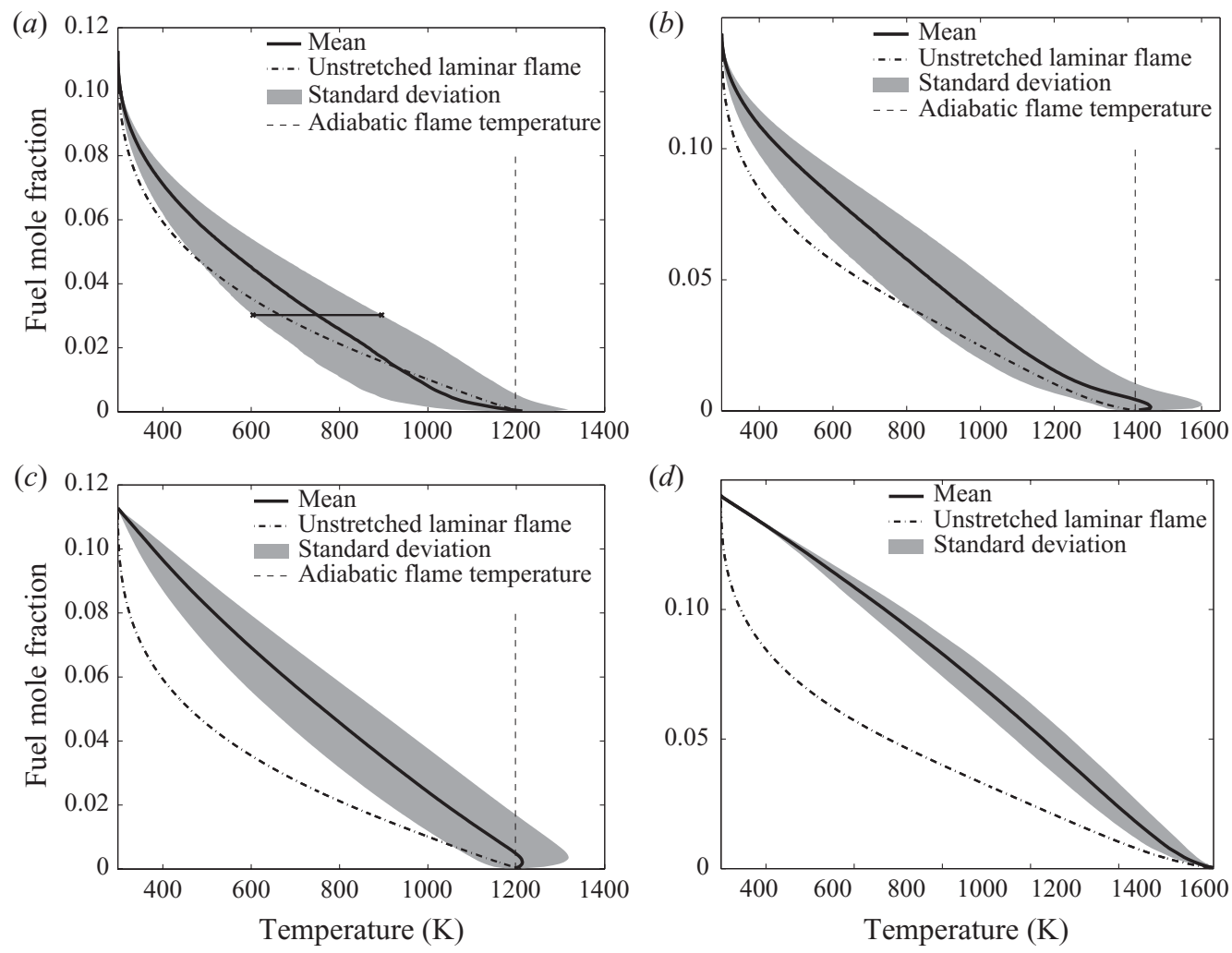

FIGURE 11. Conditional mean distribution of temperature as a function of hydrogen mole fraction. $(a-d)$ Cases A31, A40, D31 and D40, respectively. Note the mean and standard deviation are taken as a function of fuel mole fraction (as denoted in $(a)$ by the horizontal solid line). The vertical dashed line denotes the adiabatic flame temperature.

shown on the abscissa. Again, the solid curve denotes the mean equivalence ratio, the dashed curve is the distribution from the corresponding unstretched laminar flame, and the shaded region shows the standard deviation of equivalence ratio. As before, case A31 appears to follow a similar path to unstretched laminar flame, but with a broadened distribution. Using temperature as the independent variable emphasises the hot spots, which are clearly correlated with richer regions as a result of more intense burning where there is a focusing of hydrogen. The low variation in the adiabatic flame temperature arises due to the large volume of product gas in the domain. The trends here are similar to the previous figure. The richer flame (A40) again has a broader distribution, close to the corresponding unstretched laminar flame. The higher Karlovitz case (D31) is transitional, the distribution is still broad with an extended range of hot spots, but has moved away from the unstretched laminar flame distribution. Case D40, however, is again distinct. The mean has collapsed almost to a constant value, with a small standard deviation in equivalence ratio. This reinforces the increased role of turbulent mixing. If mixing is driven solely by turbulence, then hydrogen- and oxygen-containing molecules are advected together in packets, mixed at the same rate by turbulent diffusion, with little effect of differential molecular diffusion, so the equivalence ratio remains fixed. 

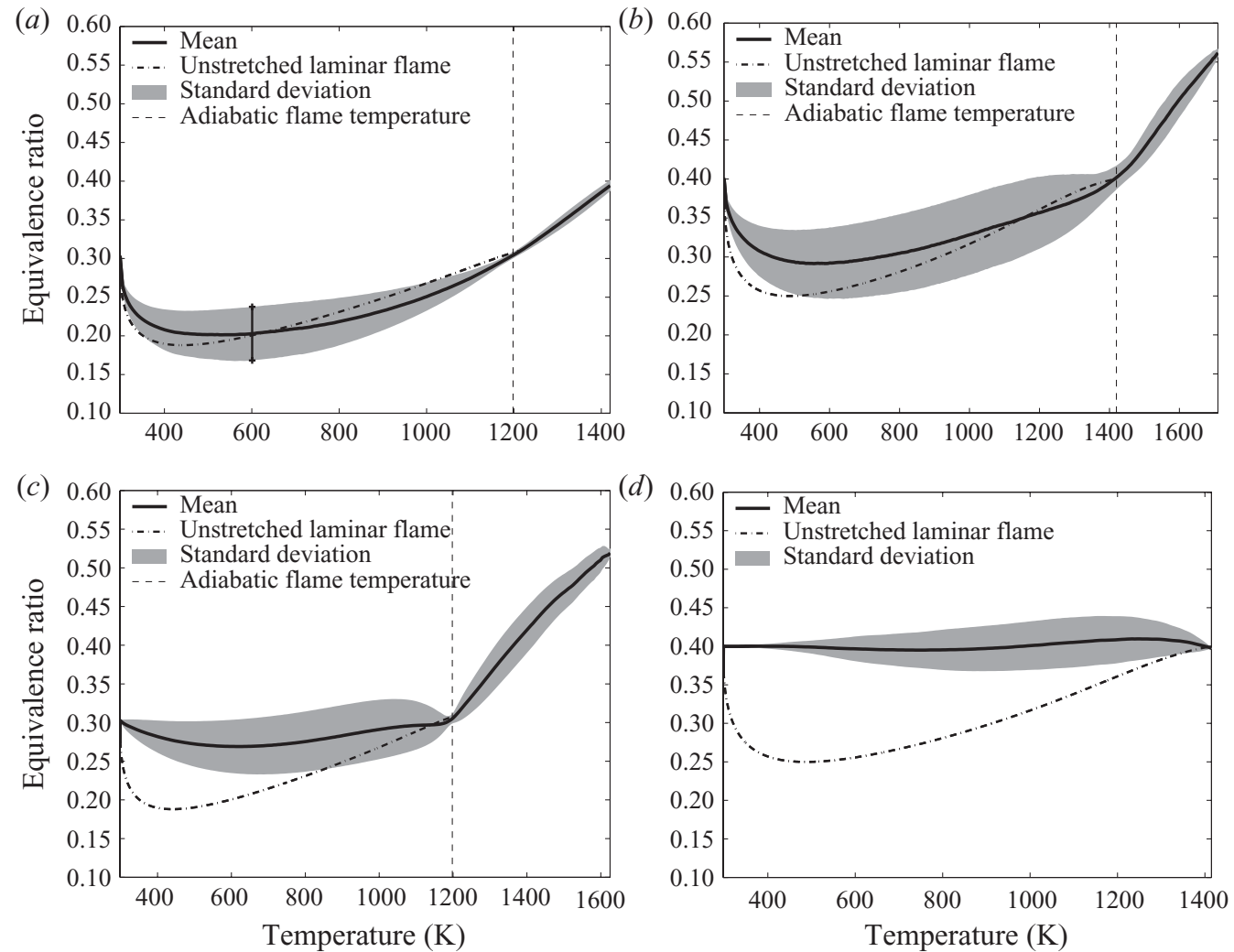

FIgURE 12. Conditional mean distribution of local equivalence ratio as a function of temperature for cases $(a-d)$ A31, A40, D31 and D40, respectively. Note the mean and standard deviation are taken as a function of temperature (as denoted in $(a)$ by the vertical solid line). The vertical dashed line denotes the adiabatic flame temperature.

\subsection{Horizontal averaging}

An alternative approach to analysing the flame structure, with more of a modelling slant, is to consider Favre-averaged quantities. The goal here is to average the flames in both space and time. The flames considered here are propagating through the domain with a speed that is statistically stationary. Therefore, to average the flames in time, we need to transform into a coordinate system that propagates with the flame. For the spatial averaging, the most natural way to average is to average horizontally, normal to the mean direction of flame propagation. We note that implicit within this approach is an ansatz that the flame is statistically flat.

\subsubsection{Averaging procedure}

To transform the solution into a flame-fixed coordinate frame, we need to identify a location of the flame as a function of time. For each point in time, we define the instantaneous global flame position by integrating the fuel mass fraction and normalising by fuel density and cross-sectional area,

$$
z_{0}(t) \equiv \frac{1}{L^{2}\left(\rho Y_{\mathrm{H}_{2}}\right)_{0}} \int_{V} \rho Y_{\mathrm{H}_{2}} \mathrm{~d} V .
$$



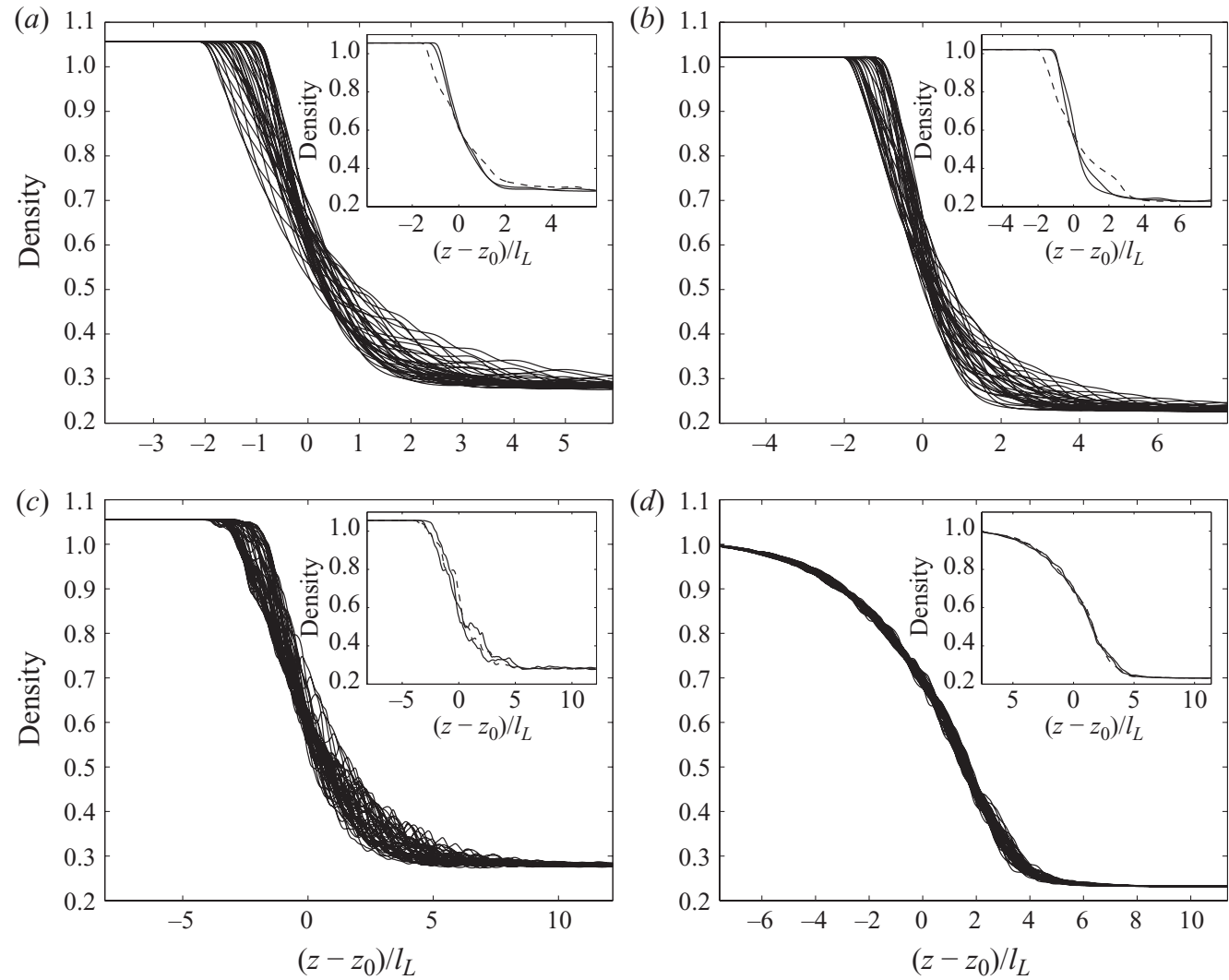

Figure 13. $(a-d)$ Density profiles for cases A31, D31, A40 and D40. Profiles are shown for all times in the averaging window, offset by the integral height $z_{0}(t)$. The insets show time dependence of the profiles; the solid lines denote the first and last profiles from the averaging window, and the dashed line is the profile of the middle time point.

Note that the turbulent flame speed is the temporal derivative of $z_{0}$, i.e. $s_{T}=\mathrm{d} z_{0} / \mathrm{d} t$. We can now define a new vertical coordinate $\zeta=z-z_{0}(t)$ that recasts the solution in frame that moves with the global flame position. This position allows for averaging to be performed over many time points as well as horizontally. In particular, for any quantity $q(x, y, z, t)$ we can define the horizontal average over the time interval $t \in\left[t_{0}, t_{1}\right]$ as

$$
\bar{q}(\zeta) \equiv \frac{1}{L^{2}\left(t_{1}-t_{0}\right)} \int_{t_{0}}^{t_{1}} \int_{0}^{L} \int_{0}^{L} q\left(x, y, z-z_{0}(t), t\right) \mathrm{d} x \mathrm{~d} y \mathrm{~d} t,
$$

where $t \in\left[t_{0}, t_{1}\right]$ is the time interval over which the flame is considered to be statistically stationary, which was shown by the horizontal lines in figure 7 for the $\varphi=0.31$ cases. The Favre average is then defined as usual $\tilde{q} \equiv \overline{\rho q} / \bar{\rho}$. Examples of the collapse of the data are shown in figure 13, where all density profiles over the averaging time are shown. There is more variation in the lower turbulence cases, but this is to be expected. The validity of the temporal averaging required that the variations in the profile are not correlated in time, i.e. the profiles are not getting gradually wider, for example. This is shown by the inset in each plot, which shows the first and last time points by solid lines, and the middle time point by a dashed 


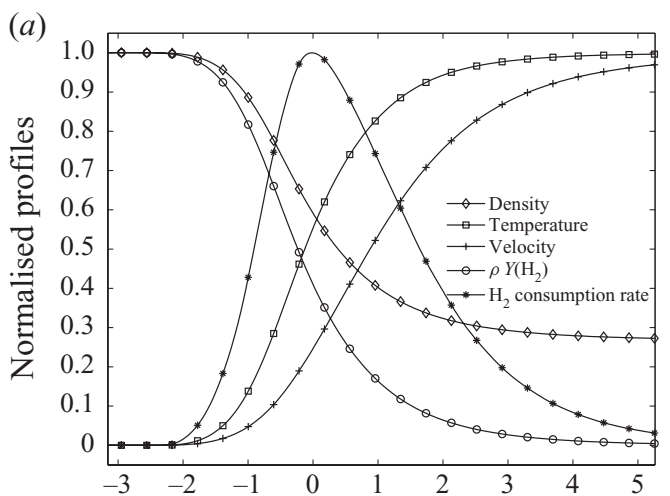

(b)
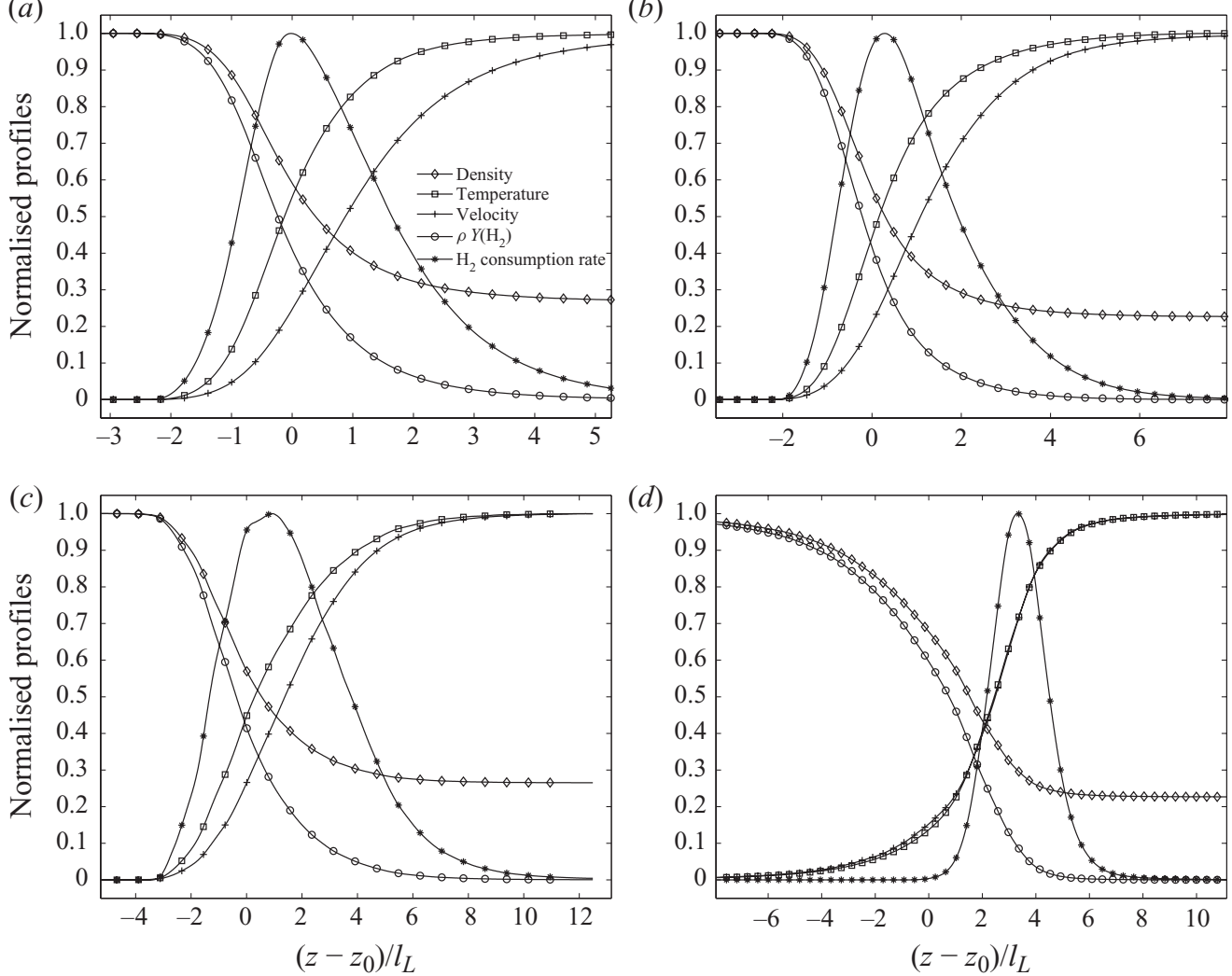

FiguRE 14. $(a-d)$ Horizontally averaged profiles for cases A31, A40, D31 and D40. The $\mathrm{H}_{2}$ consumption rate and velocity have been normalised by the corresponding peak values, and the other quantities have been normalised by the unstretched laminar flame values.

line. This demonstrates that the definition of $z_{0}$ gives a reasonable collapse of the data that is suitable for averaging, and that the data are statistically stationary over the averaging period in time.

In the remainder of the section, focus is placed on the simulations at the extremes of the parameter space explored, specifically A31, A40, D31 and D40. The results for the other cases that are not presented exhibit behaviour between these four cases.

\subsubsection{Average profiles}

Figure 14 shows normalised profiles of density, temperature, velocity, fuel density and burning rate, using the averaging procedure described above, for the four cases A31, A40, D31 and D40. Note that the markers do not correspond to computational cells; depending on the case, there are 10-40 cells between markers. The spatial ranges here have been normalized by the unstretched laminar flame thickness. Each profile has also been normalised by the corresponding unstretched laminar flame value, except the burning rate, which has been normalised by the maximum value in each turbulent case (normalising by the unstretched laminar flame value does not make sense for burning rate in the horizontally averaged framework). In general, cases A31, A40 and D31 are similar to each other in structure. The peak burning occurs close to $z_{0}$, with a longer tail downstream of the flame (also present in the profiles of fuel and density), and the velocity and temperature profiles are spatially separated. The 
(a)

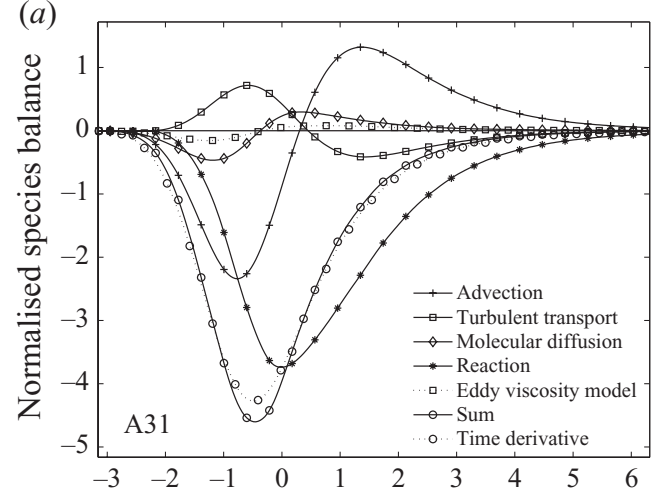

(c)

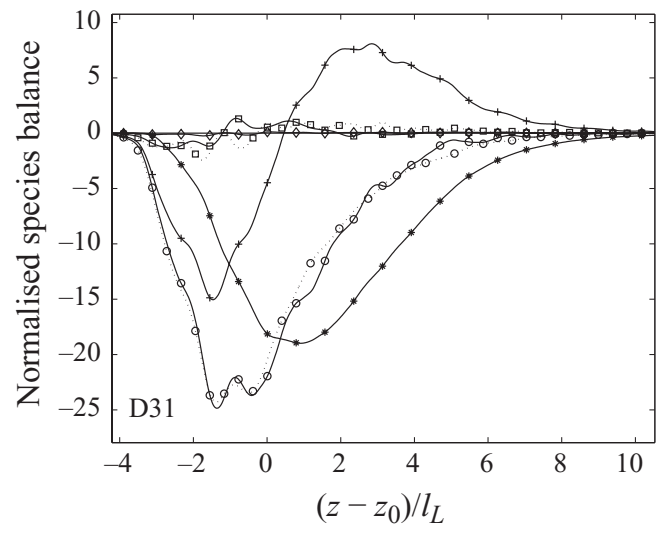

(b)

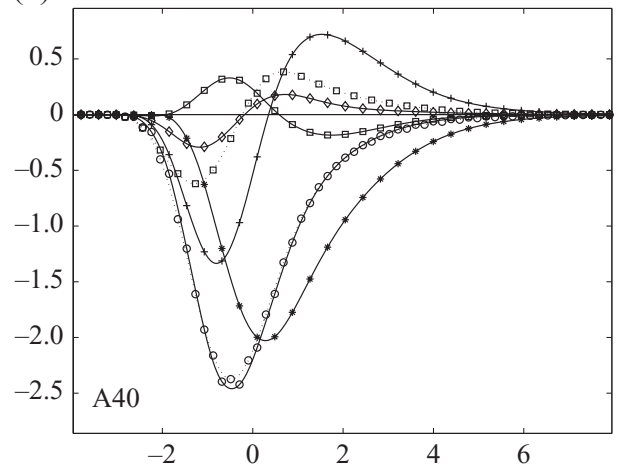

(d)

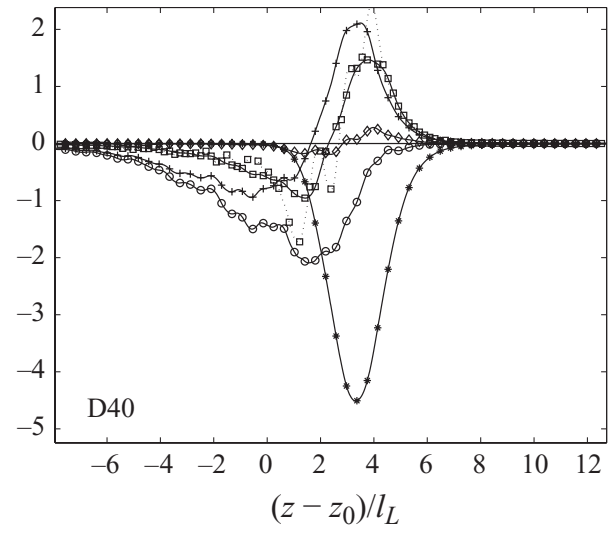

FIGURE 15. $(a-d)$ Conservation balance for $\bar{\rho} \tilde{Y}_{\mathrm{H}_{2}}$.

downstream tail is due to the inherently multi-dimensional structure of the cellular burning. Case D40 is very different. The burning occurs primarily downstream from $z_{0}$ (i.e. the burning occurs at the high-temperature end of the temperature range) and is less skewed. The profiles of density and fuel have a longer tail upstream of the flame (instead of downstream), and the velocity and temperature are no longer spatially separated. The shift in profile shape reflects the homogenisation of the mixed region in the preheat zone by turbulence.

\subsubsection{Scalar conservation balance}

To quantify the relative importance of different processes in the overall flame dynamics, we examine the contribution of different terms in Favre-averaged balance equations. First, we examine the Favre-averaged species conservation equation, which can be written as

$$
\frac{\partial}{\partial t}\left(\bar{\rho} \tilde{Y}_{i}\right)+\nabla \cdot\left(\bar{\rho} \tilde{Y}_{i} \tilde{\boldsymbol{u}}\right)=-\nabla \cdot\left(\bar{\rho} \widetilde{Y_{i}^{\prime \prime} \boldsymbol{u}^{\prime \prime}}\right)+\nabla \cdot\left(\overline{\rho D_{i} \nabla Y_{i}}\right)+\bar{\rho} \tilde{\omega}_{i},
$$

where the terms on the left-hand side are the temporal derivative and advection, and the terms on the right-hand side are the turbulent transport, molecular diffusion and reaction terms, respectively.

Figure 15 shows the Favre-averaged $\mathrm{H}_{2}$ species balance for the four extreme cases. A low-pass Gaussian filter was used to maintain smoothness in the correlations and derivatives. To check the balance, the sum of all of the terms and the temporal 
derivative are shown by solid and dotted lines with circular markers, respectively. In all cases, the mean velocity (naturally) advects $\mathrm{H}_{2}$ downstream, but the overall balance is dominated by destruction due to reaction. Again, cases A31, A40 and D31 appear similar in structure to each other. The reaction term is skewed, with a long downstream tail, whereas in case D40 it is more symmetric, but has shifted downstream. In the low-Karlovitz-number cases, molecular diffusion plays a small, but non-negligible, role, but it is almost insignificant in the high-Karlovitz-number cases.

An interesting, if unsurprising, shift is found in the turbulent transport term (solid line with square markers), which is compared with a simple eddy viscosity model (using $v_{\tau}=2 \check{u} l / 3$ ), shown by the dotted line with square markers. In the lowKarlovitz-number cases, the turbulent transport is of the opposite sign to the eddy viscosity model: counter-gradient turbulent transport is observed (see e.g. Veynante et al. 1997; Poinsot \& Veynante 2005). Much higher turbulent transport is observed in case D40, where it is comparable in magnitude to the mean advection term. Gradient diffusion is clearly evident in a distributed flame.

\subsection{Diffusion processes}

\subsubsection{Scalar fluctuation transport equation}

The persistence of the cellular burning structures observed for most of the cases (see figures 5, 8 and 10) suggests that molecular diffusion remains an important process. However, this is not captured in the averaged-species conservation equation in figure 15 because horizontal diffusion integrates to zero. To compare the effects of molecular diffusion with turbulent mixing, we consider the Favre-averaged species fluctuation conservation equation, which can be written as

$$
\begin{aligned}
\frac{\partial}{\partial t}\left(\bar{\rho} \widetilde{Y_{i}^{\prime \prime 2}}\right)+ & \nabla \cdot\left(\bar{\rho} \widetilde{Y_{i}^{\prime \prime}} \tilde{\boldsymbol{u}}\right)=-\nabla \cdot\left(\bar{\rho} \widetilde{Y_{i}^{\prime \prime 2} \boldsymbol{u}^{\prime \prime}}\right)-2\left(\bar{\rho} \widetilde{Y_{i}^{\prime \prime} \boldsymbol{u}^{\prime \prime}}\right) \cdot \nabla \tilde{Y}_{i} \\
& +\nabla \cdot\left(\overline{\rho D_{i} \nabla Y_{i}^{\prime \prime 2}}\right)+2 \overline{Y_{i}^{\prime \prime} \nabla \cdot\left(\rho D_{i} \nabla \tilde{Y}_{i}\right)}-2 \overline{\rho D\left|\nabla Y_{i}^{\prime \prime}\right|^{2}}+\bar{\rho} \widetilde{Y_{i}^{\prime \prime} \omega_{i}},
\end{aligned}
$$

where again the terms on the left-hand side are the temporal derivative and advection, and those on the right-hand side are the turbulent transport, turbulent production, molecular diffusion (two terms), molecular dissipation and reaction, respectively.

Figure 16 shows the $\mathrm{H}_{2}$ fluctuation balance for the usual four cases. The dominant balance in general is production of fluctuations by reaction and destruction by molecular dissipation. In cases A31, A40 and D31, the remaining terms (advection, turbulent production and diffusion and molecular diffusion) are about an order of magnitude smaller. A shift in behaviour is observed in case D40. The turbulent production of species fluctuations has become comparable in magnitude to the dominant balance terms (reaction and molecular dissipation), and is the main source of fluctuations. Note, in particular, that the turbulent production is negative in the other three cases. Thus, the expected transition from counter-gradient diffusion to gradient diffusion is also present in the turbulent diffusion term.

It is instructive to consider an order-of-magnitude analysis following Tennekes \& Lumley (1972) and Mantel \& Borghi (1994). Such an analysis was presented for the scalar fluctuation equation in Darbyshire, Swaminathan \& Hochgreb (2010), which demonstrated that the dominant terms were indeed production due to reaction balanced by molecular dissipation. At the next order of magnitude, it was found that turbulent production was balanced by turbulent diffusion, which is inconsistent with case D40, where turbulent production was found to be a leading-order term 


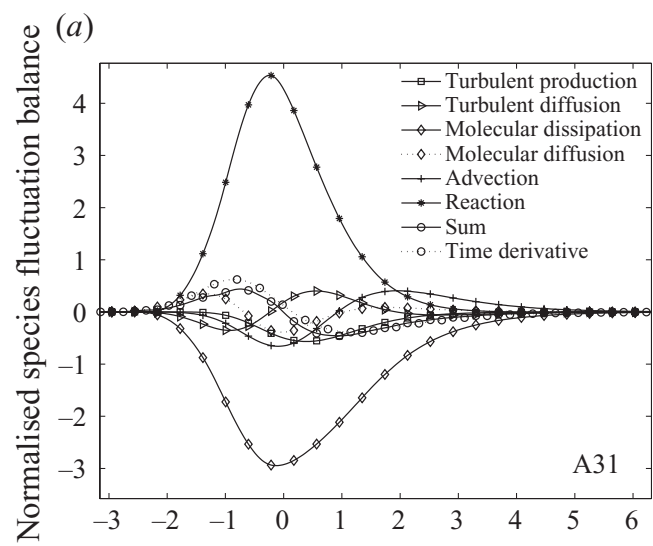

(b)
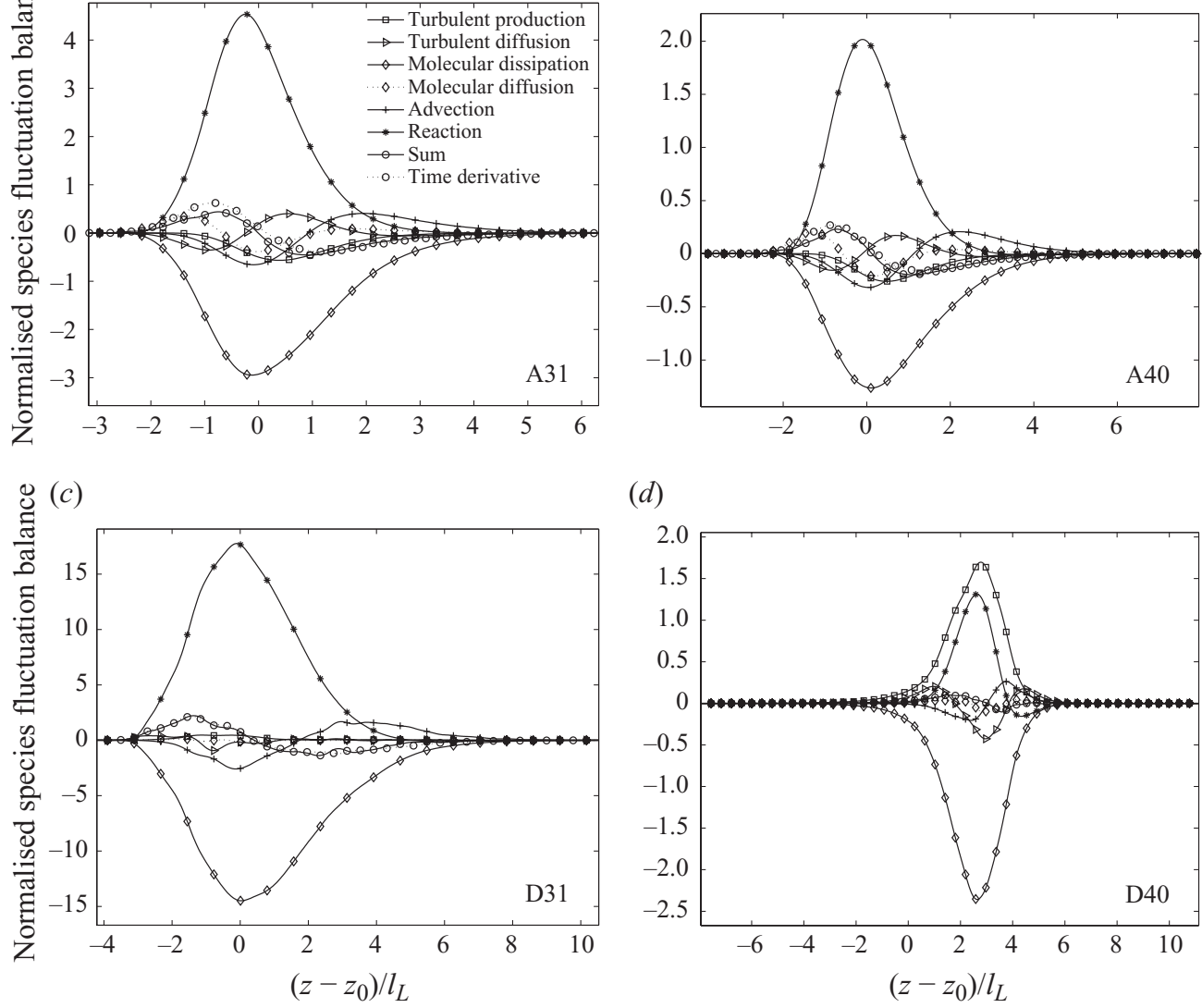

(d)

Figure 16. $(a-d)$ Conservation balance for $\bar{\rho} \widetilde{{Y_{\mathrm{H}_{2}}^{\prime \prime}}^{2}}$.

and turbulent diffusion was an order of magnitude smaller. Here, we present a slight refinement of the analysis, which is found to be consistent with the observations in both Darbyshire et al. (2010) and figure 16. The analysis also provides insight into the transition to the distributed burning regime.

Darbyshire et al. (2010) used $\rho_{u}$ to denote the density scale, derivatives of mean quantities were assumed to scale with integral length scale $l$, and derivatives of fluctuations were assumed to scale with the unstretched laminar flame width $l_{L}$. The mean and fluctuating velocities were scaled with a reference velocity $u_{\text {ref }}$ and the unstretched laminar flame speed $s_{L}$, respectively. The magnitudes of the scalar mean and fluctuations were taken to be equal and constant (which is why the turbulent production and turbulent diffusion terms were found to be of the same order of magnitude). Viscosity and scalar diffusion were assumed to scale with $s_{L} l_{L}$. Here, the mean and fluctuating velocities are both assumed to scale with $\check{u}$, and the magnitude of the scalar $Y$ and its fluctuation $\check{Y}$ are included through their ratio $\theta=\check{Y} / Y$ (note $\theta \ll 1$ ). Derivatives of fluctuations are assumed to scale with some length scale $\lambda_{Y}$, to be determined. This assumption allows the length scale at which scalar fluctuations are dissipated to vary depending on the flow. Defining $S_{r}=\rho_{u} \theta Y^{2} s_{L} / l_{L}$ (which is representative of the rate of scalar fluctuation production on the flame time scale), 
and using relations (1.1), the resulting scalings are

$$
\left.\begin{array}{rl}
\left.\widetilde{Y_{i}^{\prime \prime} \nabla \cdot\left(\rho D_{i} \nabla \tilde{Y}_{i}\right.}\right) & =O\left(S_{r} \frac{1}{R e_{L} D a_{L}}\right), \quad \nabla \cdot\left(\widetilde{\rho D_{i} \nabla Y_{i}^{\prime \prime 2}}\right)=O\left(S_{r} \frac{1}{\sqrt{R e_{L} D a_{L}}} \frac{\theta l_{L}}{\lambda_{Y}}\right), \\
\frac{\partial}{\partial t}\left(\bar{\rho} \widetilde{Y_{i}^{\prime \prime 2}}\right) & =O\left(S_{r} \frac{\theta}{D a_{L}}\right), \quad \nabla \cdot\left(\widetilde{\rho} Y_{i}^{\prime \prime 2} \tilde{\boldsymbol{u}}\right)=O\left(S_{r} \frac{\theta}{D a_{L}}\right), \\
\nabla \cdot\left(\bar{\rho} Y_{i}^{\prime \prime 2} \boldsymbol{u}^{\prime \prime}\right) & =O\left(S_{r} \frac{\theta}{D a_{L}}\right), \quad\left(\bar{\rho} \widetilde{Y_{i}^{\prime \prime} \boldsymbol{u}^{\prime \prime}}\right) \cdot \nabla \tilde{Y}_{i}=O\left(S_{r} \frac{1}{D a_{L}}\right), \\
\widetilde{\rho D_{i}\left|\nabla Y_{i}^{\prime \prime}\right|^{2}} & =O\left(S_{r} \frac{\theta l_{L}^{2}}{\lambda_{Y}^{2}}\right), \quad \bar{\rho} \widetilde{Y_{i}^{\prime \prime} \omega_{i}}=O\left(S_{r} 1\right) .
\end{array}\right\}
$$

Assuming $R e_{L} \gg 1$ and $D a_{L} \gg 1$, the above scalings are presented in increasing order of magnitude. To leading order, production of scalar fluctuations due to reaction is balanced by molecular dissipation, and the length scale of the fluctuation gradients is $\lambda_{Y} \sim l_{L} \sqrt{\theta}$. This is consistent with the analysis and data of Darbyshire et al. (2010), and the present data for all cases except D40. Also, note that only the molecular diffusion terms have Reynolds number dependency, and the turbulent transport terms do not (because the velocity fluctuation was taken to scale with $\breve{u}$ rather than $s_{L}$ ).

As the turbulent intensity increases (corresponding to the transition to the distributed burning regime), the Damköhler number decreases and the turbulent production term becomes more significant. Unlike Darbyshire et al. (2010), the turbulent diffusion remains smaller due to the inclusion of the relative magnitudes of mean and fluctuating scalars. This is consistent with case D40. Also note that this is consistent with the non-reacting case (e.g. Tennekes \& Lumley 1972), where turbulent production is balanced by molecular dissipation, and turbulent diffusion is an order of magnitude smaller. To consider the limit of small Damköhler number, the order-of-magnitude analysis can be recast in terms of $S_{p}=\rho_{u} \theta Y^{2} \breve{u} / l$ (which is representative of the rate of scalar fluctuation production on the turbulence time scale), yielding (again in increasing order of magnitude)

$$
\left.\begin{array}{rl}
\left.\widetilde{Y_{i}^{\prime \prime} \nabla \cdot\left(\rho D_{i} \nabla \tilde{Y}_{i}\right.}\right) & =O\left(S_{p} \frac{1}{R e_{L}}\right), \quad \nabla \cdot\left(\overline{\rho D_{i} \nabla Y_{i}^{\prime \prime 2}}\right)=O\left(S_{p} \sqrt{\frac{D a_{L}}{R e_{L}}} \frac{\theta l_{L}}{\lambda_{Y}}\right), \\
\bar{\rho} \widetilde{Y_{i}^{\prime \prime} \omega_{i}} & =O\left(S_{p} D a_{L}\right), \quad \frac{\partial}{\partial t}\left(\widetilde{\rho} \widetilde{Y_{i}^{\prime \prime 2}}\right)=O\left(S_{p} \theta\right), \\
\nabla \cdot\left(\bar{\rho} \widetilde{Y_{i}^{\prime \prime 2}} \tilde{\boldsymbol{u}}\right) & =O\left(S_{p} \theta\right), \quad \nabla \cdot\left(\bar{\rho} \widetilde{Y_{i}^{\prime \prime 2} \boldsymbol{u}^{\prime \prime}}\right)=O\left(S_{p} \theta\right), \\
\overline{\rho D_{i}\left|\nabla Y_{i}^{\prime \prime}\right|^{2}} & =O\left(S_{p} \frac{l_{L}^{2} \theta D a_{L}}{\lambda_{Y}^{2}}\right), \quad\left(\bar{\rho} \widetilde{Y_{i}^{\prime \prime} \boldsymbol{u}^{\prime \prime}}\right) \cdot \nabla \tilde{Y}_{i}=O\left(S_{p} 1\right) .
\end{array}\right\}
$$

In this form, the reaction term becomes small, and turbulent production becomes the leading-order term balanced by molecular dissipation, with $\lambda_{Y} \sim l_{L} \sqrt{\theta D a_{L}}$. This describes the limiting behaviour of the distributed burning regime and is independent of the properties of the fuel.

\section{Discussion and conclusions}

We have presented a comprehensive study of the effects of turbulence on lean premixed hydrogen flames in an idealised setting. Under these conditions, the flames 
are thermodiffusively unstable and it is impossible to stabilise an unstretched steady laminar flame. Furthermore, the conventional characterisation of turbulent flames in this regime by Karlovitz and Damköhler numbers based on these non-physical unstretched laminar flame properties undermines the universality of these parameters across a range of equivalence ratios. Specifically, in more than one dimension, preferential diffusion of hydrogen leads to local enrichment and enhanced burning, which was found to have a disproportionately greater effect at lower equivalence ratios. We speculate that modifying these dimensionless numbers to be defined in terms of freely propagating three-dimensional flames (without turbulence) will lead to a more universal characterisation, and will be the subject of future work.

At an equivalence ratio of $\varphi=0.31$, increasing levels of turbulence resulted in increased peak local burning rate and overall turbulent flame speeds, the cellular structure became increasingly disrupted and at a Karlovitz number of 1560, the flame became a broad distribution of small pockets of intense burning. A similar response was found for low-to-moderate Karlovitz numbers at an equivalence ratio of $\varphi=0.40$, but a categorically different behaviour was observed at $K a_{L}=1560$ : a distributed flame, the consequences of which will be discussed in detail below. The persistence of the cellular burning structure meant that more intense turbulence was required to produce a distributed flame than in the supernova case, but we suspect that the higher density ratio in the present case is another contributing factor.

Under the conditions studied, global extinction was not observed and the turbulent flame speeds were all faster than the corresponding unstretched laminar flame speeds.

\subsection{What is distributed burning?}

We consider the distributed burning regime to be the limiting behaviour of flame propagation where turbulence is responsible for the mixing of fuel, oxidiser and heat, dominating the thermal and species diffusion that are responsible for flame propagation at lower turbulent intensities. For a particular fuel, a critical Karlovitz number is required; the turbulent time scales must be shorter than chemical time scales. This critical Karlovitz number may be determined by requiring the Kolmogorov length scale to be smaller than the reaction zone thickness of the flame $\eta<\delta$, and hence $K a_{c}=K a_{\delta}$, but may depend on other factors, including Lewis number or the density jump across the flame (a large density jump will lead to a pronounced expansion of the fluid and suppression of the turbulence, requiring higher Karlovitz numbers to distribute the reaction zone). Our characterisation of the distributed burning regime coincides with the broken reaction zone of Peters $(1999,2000)$, where exposure to high levels of stretch and/or a cold ambient fluid is responsible for global extinction of the flame. We contend that both kinds of flames can exist and will depend on the configuration of the flow. We note that our observations are consistent with the descriptions of distributed burning according to Summerfield et al. (1954, 1955), Williams (1985a), Bray (1995) and Pope \& Anand (1985).

In this paper, we have presented lean premixed hydrogen flames over a range of Karlovitz numbers, including a flame burning in the distributed mode. Several diagnostics were presented to demonstrate the increased role of turbulent mixing, and characterised several properties of distributed flames. In a distributed flame, there is no longer a sharp interface between the fuel and products; a broad mixed 
region in the density that resembles a turbulent mixing zone forms at the leading edge of the flame before any significant reactions occur. The normalised PDF of $|\nabla \rho|$ was found to tend towards an exponential distribution as the flame became distributed, characteristic of turbulent scalar mixing. For the present hydrogen flames at low Karlovitz numbers, a long tail was observed downstream of the flame and the burning rate was skewed. In the distributed flame, the burning rate appeared to be relatively symmetric and the tail in the other profiles were upstream of the flame. The burning was observed to move downstream to the high-temperature end of the distribution, and was lower in intensity than at lower Karlovitz numbers, but higher than the corresponding unstretched laminar flame.

The dominance of turbulent mixing has several consequences. First, the expected transition from counter-gradient diffusion to gradient diffusion was observed with the transition to distributed burning. In the distributed regime, the turbulent diffusion coefficient is large compared with molecular diffusion coefficients, which means a simple eddy viscosity model approach is an appropriate turbulence model for distributed flames. This also means that the distributed limit will be independent of fuel, including larger hydrocarbons, although the critical Karlovitz number required is expected to vary. In the absence of reactions, the flow can be considered to be a two-fluid mixing problem. Fuel and products are materially advected until mixed at small scales, and so each volume of fluid is a linear combination of pure fuel and pure product. In essence, reactions merely limit the thickness of the mixing zone. This means that the effective Lewis number of each species is effectively unity, and so there is little change in local equivalence ratio. Also, this results in the collapse of JPDFs such as the fuel-temperature conditional mean shown in figure 11. Quantities such as density and temperature cannot exceed the extrema of pure fuel or pure product. This was particularly relevant in the temperature field, where there was an absence of hot spots found at lower Karlovitz numbers, which has the potential for reduced thermal $\mathrm{NO}_{x}$ emissions.

This work was supported by the Applied Mathematics Research Program of the US Department of Energy. A.J.A. was also supported by a Glenn T. Seaborg Fellowship. Simulations were performed on the Lawrencium computational cluster resource provided by the IT Division at the Lawrence Berkeley National Laboratory, and high-resolution simulations on Franklin (under an Incite award) and Hopper at National Energy Research Scientific Computing Center. All support is provided by the US Department of Energy under contract DE-AC02-05CH11231.

\section{Appendix A. Convergence study}

The algorithm used for the present study is based on a non-oscillatory finite-volume approach that remains stable even when under-resolved, and so some care is required to ensure that simulations are well-resolved. It was shown in Aspden et al. (2008b) that a marginally resolved viscous simulation has an effective Kolmogorov length scale and effective viscosity that is slightly larger than the physical value. Dimensional analysis was used to characterise the inviscid extreme, known as implicit large-eddy simulation (ILES), through an analogy with the theory of Kolmogorov (1941). From this analysis, an expression for the effective viscosity $v_{e}$ was obtained that describes the transition from well-resolved to pure ILES, $v_{e}=v_{u}+v_{\Delta x} \exp \left(-v_{u} / 2 v_{\Delta x}\right)$, from which the effective Kolmogorov length scale can be derived ( $v_{\Delta x}$ is the effective viscosity of 

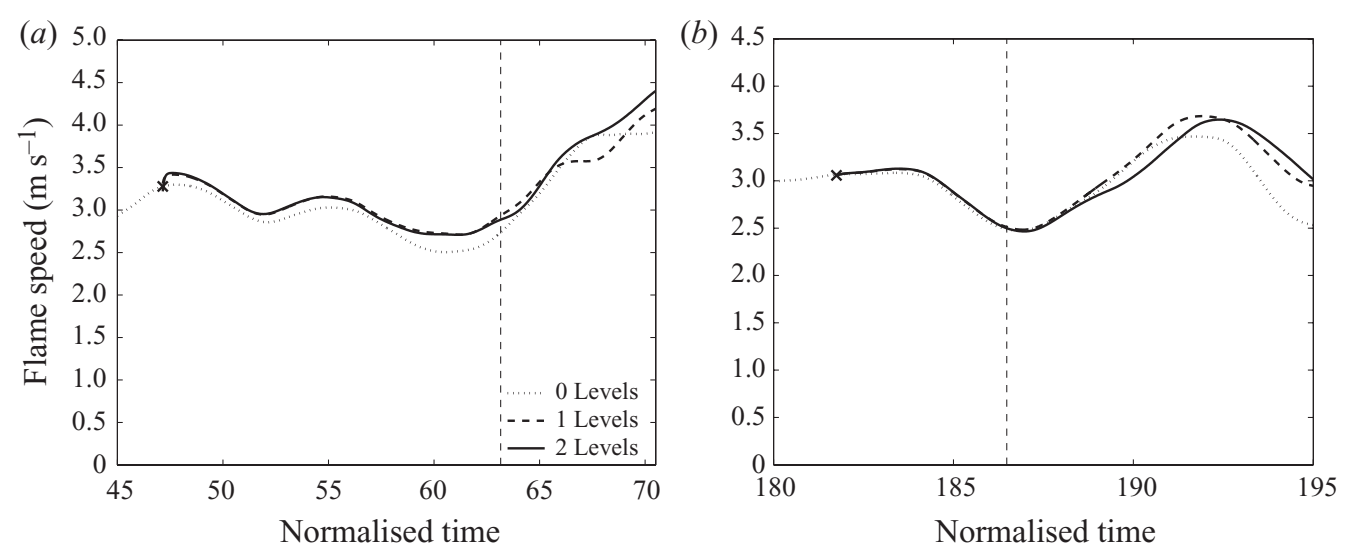

FIGURE 17. Turbulent flame speeds demonstrating convergence for (a) C31 and (b) D40. Dashed black lines denote times of the slices in figures 18 and 19.

a simulation with a cell width $\Delta x$ and zero diffusion, and $v_{u}$ is the specified physical viscosity).

To demonstrate that the simulations are sufficiently well-resolved, we present a convergence analysis for two of the key cases. Specifically, cases C31 and D40 are considered because the chemistry in case $\mathrm{C} 31$ is challenging at the resolution presented, and D40 is the key simulation of a distributed flame. In all of the simulations presented, a low-resolution simulation was run to establish the flame, and then the adaptive mesh refinement was turned on to add the necessary resolution for the statistically steady part of the evolution. Here, we present three simulations for each of the two cases, beginning at the point where the AMR is turned on. The three simulations are at three different resolutions, which was achieved by running without AMR, one level of AMR, and two levels of AMR. This gives effective resolutions of $64 \times 64 \times 512,128 \times 128 \times 1024$ and $256 \times 256 \times 2048$, respectively.

Figure 17 shows the turbulent flame speeds for the six simulations, the time scale has been normalised by the integral length-scale turnover time. The dotted, dashed and solid lines denote the $64 \times 64 \times 512,128 \times 128 \times 1024$ and $256 \times 256 \times 2048$ simulations, respectively. The cross denotes the time when the AMR was added. In case $\mathrm{C} 31$, the two higher-resolution cases depart immediately from the low-resolution case, indicating that the $64 \times 64 \times 512$ resolution is insufficient in this case, but are in close agreement with each other for over 15 large-scale eddy turnover times. The turbulent nature of the flow makes it inherently unstable, and to match the turbulent flame speed for so many turnover times demonstrates that the turbulence-flame interactions are being sufficiently well-resolved. The $128 \times 128 \times 1024$ simulation was used for case C31. In case D40, all three simulations are in close agreement for five turnover times, but due to the higher levels of turbulence, deviation was found to occur relatively earlier. The $256 \times 256 \times 2048$ simulation was used in this case to ensure that the turbulence was well-resolved.

Figure 18 shows slices of case $\mathrm{C} 31$ at the three resolutions at the time indicated by the vertical dashed line in figure $17(a)$. Even after so many eddy turnover times, the structure of the flame is clearly still very similar. Figure 19 shows slices of case D40 at the three resolutions at the time indicated by the vertical dashed line in figure $17(b)$. Again, the structure of the flame in each case is very similar. 


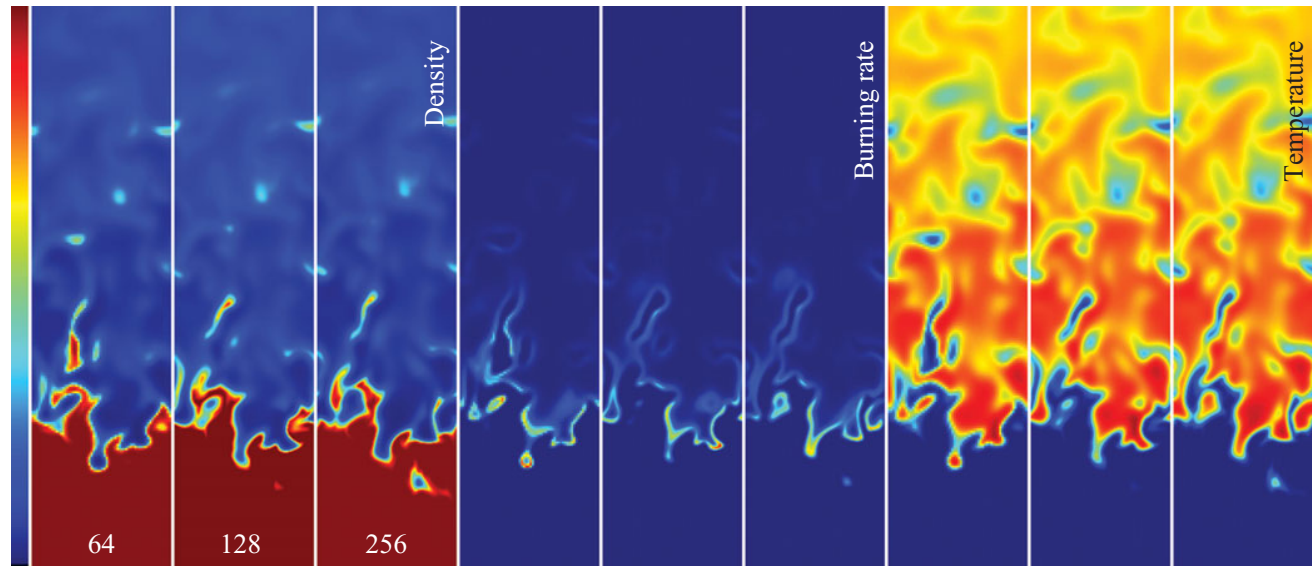

FIGURE 18. Two-dimensional slices of density, burning rate and temperature for case C31 at three different resolutions.

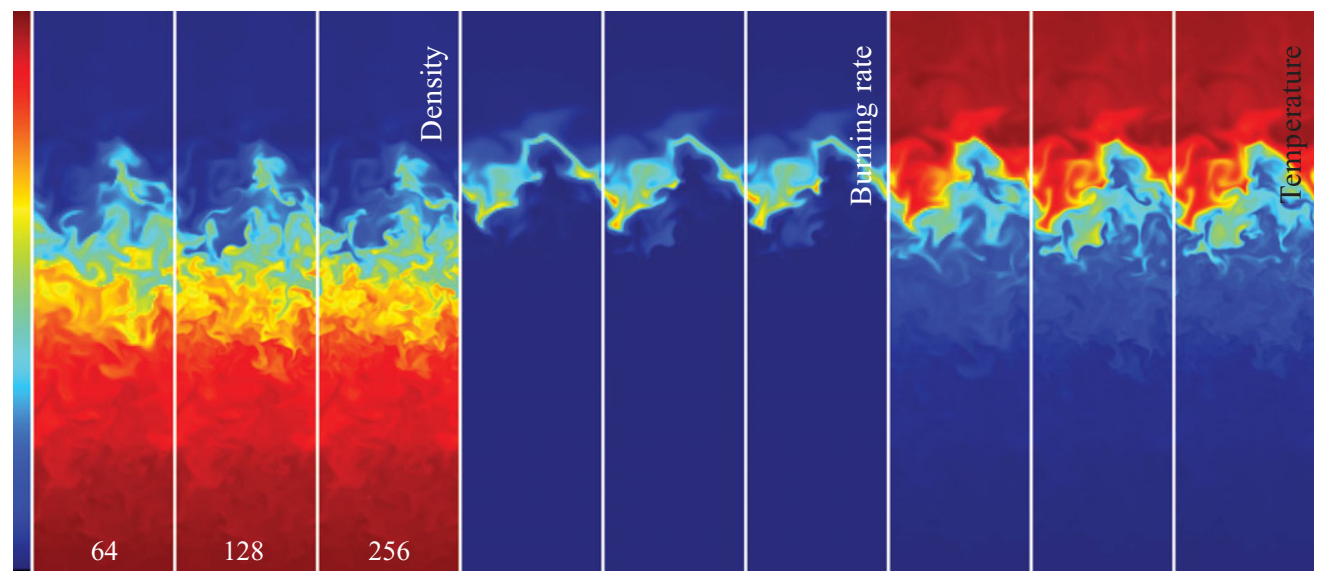

FIGURE 19. Two-dimensional slices of density, burning rate and temperature for case D40 at three different resolutions.

\section{REFERENCES}

Abraham, J., Williams, F. A. \& Bracco, F. V. 1985 A discussion of turbulent flame structure in premixed charges. SAE 850345.

Almgren, A. S., Bell, J. B. \& Crutchfield, W. Y. 2000 Approximate projection methods: Part I. Inviscid analysis. SIAM J. Sci. Comput. 22, 1139-1159.

Almgren, A. S., Bell, J. B. \& SzymczaK, W. G. 1996 A Numerical Method for the Incompressible Navier-Stokes Equations Based on an Approximate Projection. SIAM J. Sci. Comput. 17, 358-369.

Aspden, A. J., Bell, J. B., Day, M. S., Woosley, S. E. \& Zingale, M. $2008 a$ Turbulence-Flame Interactions in Type Ia Supernovae. Astrophys. J. 689, 1173-1185.

Aspden, A. J., Bell, J. B. \& Woosley, S. E. 2010 Distributed Flames in Type Ia Supernovae. Astrophys. J. 710, 1654-1663.

Aspden, A. J., Nikiforakis, N., Dalziel, S. B. \& Bell, J. B. $2008 b$ Analysis of Implicit LeS methods. Commun. Appl. Math. Comput. Sci. 3 (1), 103-126. 
Baum, M., Poinsot, T. J., Haworth, D. C. \& Darabiha, N. 1994 Direct numerical simulation of $\mathrm{H}_{2} / \mathrm{O}_{2} / \mathrm{N}_{2}$ flames with complex chemistry in two-dimensional turbulent flows. J. Fluid Mech. 281, $1-32$.

Bell, J. B., Brown, N. J., Day, M. S., Frenklach, M., Grcar, J. F. \& Tonse, S. R. 2000 The dependence of chemistry on the inlet equivalence ratio in vortex-flame interactions. Proc. Combust. Inst. 28, 1933-1939.

Bell, J. B., Cheng, R. K., Day, M. S., Beckner, V. E. \& Lijewski, M. J. 2008 Interaction of turbulence and chemistry in a low-swirl burner. J. Phys.: Conf. Ser. 125, 012027.

Bell, J. B., Cheng, R. K., Day, M. S. \& Shepherd, I. G. 2007 a Numerical simulation of Lewis number effects on lean premixed turbulent flames. Proc. Combust. Inst. 31, 1309-1317.

Bell, J. B., DAY, M. S. \& GrCAR, J. F. $2002 a$ Numerical simulation of premixed turbulent methane combustion. Proc. Combust. Inst. 29, 1987-1993.

Bell, J. B., Day, M. S., Grcar, J. F., Bessler, W. G., Schultz, C., Glarborg, P. \& Jensen, A. D. $2002 b$ Detailed modeling and laser-induced fluorescence imaging of nitric oxide in a $\mathrm{NH}_{3}$-seeded non-premixed methane/air flame. Proc. Combust. Inst. 29, 2195-2202.

Bell, J. B., Day, M. S., Grcar, J. F., Lijewski, M. J., Driscoll, J. F. \& Filatyev, S. F. $2007 b$ Numerical simulation of a laboratory-scale turbulent slot flame. Proc. Combust. Inst. 31, 1299-1307.

Bell, J. B., Day, M. S., Shepherd, I. G., Johnson, M., Cheng, R. K., Grcar, J. F., Beckner, V. E. \& LiJewski, M. J. 2005 Numerical simulation of a laboratory-scale turbulent V-flame. Proc. Natl Acad. Sci. USA 102 (29), 10006-10011.

Borghi, R. 1985 On the structure of turbulent premixed flames. In Recent Advances in Aeronautical Science (ed. C. Bruno \& C. Casci). Pergamon.

Bowman, C. T., Hanson, R. K., Davidson, D. F., Gardiner, W. C. JR, Lissianski, V., Smith, G. P., Golden, D. M., Frenklach, M. \& GoldenberG, M. 1995 GRI-Mech: an optimized detailed chemical reaction mechanism for methane combustion. Available at: http://www.me. berkeley.edu/gri_mech/.

Bray, K. N. C. 1995 Turbulent Transport in Flames. R. Soc. Proc. Lon. A 451, 231-256.

Bregeon, B., Gordon, A. S. \& Williams, F. A. 1978 Near-limit downward propagation of hydrogen and methane flames in oxygen/nitrogen mixtures. Combust. Flame 33, 33-45.

Bremer, P.-T., Weber, G., Tierny, J., Pascucci, V., Day, M. \& Bell, J. 2009 A topological framework for the interactive exploration of large scale turbulent combustion. In Proc. 5th IEEE Intl Conf. on e-Science, pp. 247-254.

Chen, Y.-C. \& Mansour, M. S. 1997 Simultaneous Rayleigh scattering and laser-induced CH fluorescence for reaction zone imaging in high-speed premixed hydrocarbon flames. Appl. Phys. B 64 (5), 599-605.

Chen, Y.-C., Peters, N., Schneemann, G. A., Wruck, N., Renz, U. \& Mansour, M. S. 1996 The detailed flame structure of highly stretched turbulent premixed methane-air flames. Combust. Flame 107 (3), 223-244.

DAmKöHLER, G. 1940 Der Einfluss der Turbulenz auf die Flammengeschwindigenkeit in Gasgemischen. Z. Elektrochem. 46, 601-652.

Darbyshire, O. R., Swaminathan, N. \& Hochgreb, S. 2010 The effects of small-scale mixing models on the prediction of turbulent premixed and stratified combustion. Combust. Sci. Technol. 182, 1141-1170.

DAY, M. S. \& Bell, J. B. 2000 Numerical simulation of laminar reacting flows with complex chemistry. Combust. Theor. Model. 4, 535-556.

Day, M. S., Bell, J. B., Bremer, P.-T., Pascucci, V., Beckner, V. \& Lijewski, M. J. $2009 a$ Turbulence effects on cellular burning structures in lean premixed hydrogen flames. Combust. Flame 156 (5), 1035-1045.

Day, M. S., Bell, J. B., Cheng, R. K., Tachibana, S., Beckner, V. E. \& Lijewski, M. J. $2009 b$ Cellular burning in lean premixed turbulent hydrogen-air flames: coupling experimental and computational analysis at the laboratory scale, J. Phys.: Conf. Ser. 180, 012031.

Dunn, M. J., MasRi, A. R. \& Bilger, R. B. 2007 A new piloted premixed jet burner to study strong finite-rate chemistry effects. Combust. Flame 151 (1-2), 46-60.

Dunn, M. J., Masri, A. R., Bilger, R. W., BARlow, R. S. \& WANG, G.-H. 2009 The compositional structure of highly turbulent piloted premixed flames issuing into a hot coflow. Proc. Combust. Inst. 32 (2), 1779-1786. 
Ern, A. \& Giovangigli, V. 1994 Multicomponent Transport Algorithms. Lecture Notes in Physics, vol. m24. Springer.

Gollub, J. P., Clarke, J., Gharib, M., Lane, B. \& Mesquita, O. N. 1991 Fluctuations and transport in a stirred fluid with a mean gradient. Phys. Rev. Lett. 67 (25), 3507-3510.

JAYESH \& WARHAFT, Z. 1991 Probability distribution of a passive scalar in grid-generated turbulence. Phys. Rev. Lett. 67 (25), 3503-3506.

Kalghatgi, G. T., Cousins, J. M. \& Bray, K. N. C. 1981 Crossed beam correlation measurements and model predictions in a rocket exhaust plume. Combust. Flame 43, 51-67.

Kee, R. J., Grcar, J. F., Smooke, M. D. \& Miller, J. A. 1983 PREMiX: A Fortran program for modeling steady, laminar, one-dimensional premixed flames. Tech. Rep. SAND85-8240. Sandia National Laboratories, Livermore.

Klimov, A. M. 1963 Laminar flame in a turbulent flow. Zhur. Prikl. Mekh. Tekh. Fiz. 3, 49-58.

Kolmogorov, A. 1941 The Local Structure of Turbulence in Incompressible Viscous Fluid for Very Large Reynolds Numbers. Akad. Nauk SSSR Dokl. 30, 301-305.

Littlejohn, D. \& Cheng, R. K. 2007 Fuel effects on a low-swirl injector for lean premixed gas turbines. Proc. Combust. Inst. 31 (2), 3155-3162.

Majda, A. \& Sethian, J. A. 1985 The derivation and numerical solution of the equations for zero Mach number combustion. Combust. Sci. Technol. 42, 185-205.

Mansour, M. S., Chen, Y.-C. \& Peters, N. 1992 The Reaction Zone Structure of Turbulent Premixed Methane-Helium-Air Flames near Extinction. In 24th Symp. (International) on Combustion, pp. 461-468.

Mansour, M. S., Peters, N. \& Chen, Y.-C. 1998 Investigation of scalar mixing in the thin reaction zones regime using a simultaneous CH-LIF/Rayleigh laser technique. Proc. Combust. Inst. 27 (1), 767-773.

MANtel, T. \& Borghi, R. 1994 A new model of premixed wrinkled flame propagation based on a scalar dissipation equation. Combust. Flame 96, 443-457.

Markstein, G. H. 1949 Cell structure of propane flames burning in tubes. J. Chem. Phys. 17 (4), 428-429.

Meneveau, C. \& Poinsot, T. 1991 Stretching and quenching of flamelets in premixed turbulent combustion. Combust. Flame 86 (4), 311-332.

Mitani, T. \& Williams, F. A. 1980 Studies of cellular flames in hydrogen-oxygen-nitrogen mixtures. Combust. Flame 39, 169-190.

Pember, R. B., Bell, J. B., Colella, P., Crutchfield, W. Y. \& Welcome, M. W. 1995 An adaptive Cartesian grid method for unsteady compressible flow in irregular regions. J. Comput. Phys. 120, 278-304.

Peters, N. 1986 Laminar flamelet concepts in turbulent combustion. In 21st Symp. (International) on Combustion, pp. 1231-1250. Combustion Institute.

Peters, N. 1999 The turbulent burning velocity for large-scale and small-scale turbulence. J. Fluid Mech. 384, 107-132.

Peters, N. 2000 Turbulent Combustion. Cambridge University Press.

Poinsot, T. \& Veynante, D. 2005 Theoretical and Numerical Combustion, 2nd edn. Edwards.

Poinsot, T., Veynante, D. \& Candel, S. 1990 Diagrams of premixed turbulent combustion based on direct simulation. Proc. Combust. Inst. 23, 613-619.

Poinsot, T., Veynante, D. \& Candel, S. 1991 Quenching processes and premixed turbulent combustion diagrams. J. Fluid Mech. 228, 561-606.

Poludnenko, A. Y. \& ORAn, E. S. $2010 a$ The interaction of high-speed turbulence with flames: Global properties and internal flame structure. Combust. Flame 157, 995-1011.

Poludnenko, A. Y. \& Oran, E. S. $2010 b$ The interaction of high-speed turbulence with flames: Turbulent flame speed. Combust. Flame 158, 301-326.

Pope, S. B. 1987 Turbulent premixed flames. Annu. Rev. Fluid Mech. 19 (1), 237-270.

Pope, S. B. \& ANAND, M. S. 1985 Flamelet and distributed combustion in premixed turbulent flames. Proc. Combust. Inst. 20 (1), 403-410.

Rehm, R. G. \& Baum, H. R. 1978 The equations of motion for thermally driven buoyant flows. J. Res. Natl Bur. Stand. 83, 297-308.

Roberts, W. L., Driscoll, J. F., Drake, M. C. \& Goss, L. P. 1993 Images of the quenching of a flame by a vortex - to quantify regimes of turbulent combustion. Combust. Flame 94 (1), $58-69$. 
Strakey, P., Sidwell, T. \& OntKo, J. 2007 Investigation of the effects of hydrogen addition on lean extinction in a swirl stabilized combustor. Proc. Combust. Inst. 31, 3173-3180.

Sullivan, N., Jensen, A., Glarborg, P., Day, M. S., Grcar, J. F., Bell, J. B., Pope, C. \& Kee, R. J. 2002 Ammonia conversion and $\mathrm{NO}_{x}$ formation in laminar coflowing nonpremixed methane-air flames. Combust. Flame 131, 285-298.

Summerfield, M., Reiter, S. H., Kebely, V. \& Mascolo, R. W. 1954 The physical structure of turbulent flames. J. Jet Propul. 24 (4), 254-255.

Summerfield, M., Reiter, S. H., Kebely, V. \& Mascolo, R. W. 1955 The structure and propagation mechanism of turbulent flames in high speed flow. J. Jet Propul. 25 (8), 377-384.

Tennekes, H. \& Lumley, J. L. 1972 A First Course in Turbulence. MIT Press.

Thoroddsen, S. T. \& VAn Atta, C. W. 1992 Exponential tails and skewness of density-gradient probability density functions in stably stratified turbulence. J. Fluid Mech. 244, 547-566.

Veynante, D., Trouvé, A., Bray, K. N. C. \& Mantel, T. 1997 Gradient and counter-gradient scalar transport in turbulent premixed flames. J. Fluid Mech. 332, 263-293.

Williams, F. A. 1976 Criteria for existence of wrinkled laminar flame structure of turbulent premixed flames. Combust. Flame 26, 269-270.

Williams, F. A. 1985 a Combustion Theory. Addison-Wesley.

Williams, F. A. $1985 b$ Turbulent combustion. In Mathematics of Combustion (ed. J. Buckmaster). SIAM.

Zeldovich, Y. B. 1944 Theory of Combustion and Detonation in Gases (in Russian). Acad. Sci. USSR. 